\title{
KEPLER-18b, c, AND d: A SYSTEM OF THREE PLANETS CONFIRMED BY TRANSIT TIMING VARIATIONS, LIGHT CURVE VALIDATION, WARM-SPITZER PHOTOMETRY, AND RADIAL VELOCITY MEASUREMENTS*
}

\section{William D. Cochran ${ }^{1}$, Daniel C. Fabrycky ${ }^{2}$, Guillermo Torres ${ }^{3}$, François Fressin ${ }^{3}$, Jean-Michel Désert ${ }^{3}$, Darin Ragozzine ${ }^{3}$, Dimitar Sasselov ${ }^{3}$, Jonathan J. Fortney ${ }^{2}$, Jason F. Rowe ${ }^{4}$, Erik J. Brugamyer ${ }^{5}$, Stephen T. Bryson ${ }^{4}$, Joshua A. Carter ${ }^{3}$, David R. Ciardi ${ }^{6}$, Steve B. Howell ${ }^{4}$, Jason H. StefFen ${ }^{7}$,} William. J. Borucki ${ }^{4}$, David G. $\mathrm{KoCh}^{4}$, Joshua N. WinN ${ }^{8}$, William F. Welsh ${ }^{9}$, Kamal Uddin ${ }^{4}, 10$, Peter Tenenbaum ${ }^{4,11}$, M. Still ${ }^{4,12}$, Sara Seager ${ }^{8}$, Samuel N. Quinn ${ }^{3}$, F. Mullally ${ }^{4,11}$, Neil Miller $^{2}$, Geoffrey W. Marcy ${ }^{13}$,

Phillip J. MacQueen ${ }^{1}$, Phillip Lucas ${ }^{14}$, JaCK J. Lissauer ${ }^{4}$, David W. Latham ${ }^{3}$, Heather KNutson ${ }^{13}$, K. KinemuChi $^{4,12}$, John A. Johnson ${ }^{15}$, Jon M. Jenkins ${ }^{4}, 11$, Howard IsaAcson ${ }^{13}$, Andrew Howard ${ }^{13}$, Elliott Horch ${ }^{16}$, Matthew J. Holman ${ }^{3}$, Christopher E. Henze ${ }^{4}$, Michael R. HaAs ${ }^{4}$, Ronald L. Gilliland ${ }^{17}$, Thomas N. Gautier III ${ }^{18}$, Eric B. Ford ${ }^{19}$, Debra A. Fischer ${ }^{20}$, Mark Everett ${ }^{21}$, Michael EndL ${ }^{1}$, Brice-Oliver Demory ${ }^{8}$, Drake Deming ${ }^{22}$, David Charbonneau ${ }^{3}$, Douglas Caldwell ${ }^{4,11}$, Lars Buchinave ${ }^{23,24}$, Timothy M. Brown ${ }^{25}$, and Natalie Batalha ${ }^{26}$

${ }^{1}$ McDonald Observatory, The University of Texas, Austin, TX 78712, USA; wdc@astro.as.utexas.edu

2 Department of Astronomy and Astrophysics, University of California, Santa Cruz, CA 95064, USA

${ }^{3}$ Harvard-Smithsonian Center for Astrophysics, Cambridge, MA 02138, USA; jdesert@cfa.harvard.edu ${ }^{4}$ NASA Ames Research Center, Moffett Field, CA 94035, USA

${ }^{5}$ Department of Astronomy, The University of Texas, Austin, TX 78712, USA

${ }^{6}$ NASA Exoplanet Science Institute/California Institute of Technology, Pasadena, CA 91125, USA

${ }^{7}$ Fermilab Center for Particle Astrophysics, Batavia, IL 60510, USA

${ }^{8}$ Department of Physics, Massachusetts Institute of Technology, Cambridge, MA 02139, USA; jwinn@mit.edu, seager@mit.edu

${ }^{9}$ Department of Astronomy, San Diego State University, San Diego, CA 92182, USA; wfw@kublai.sdsu.edu

${ }^{10}$ Orbital Sciences Corporation/NASA Ames Research Center, Moffett Field, CA 94035, USA; kamal.uddin@ nasa.gov

${ }^{11}$ ETI Institute, Mountain View, CA, 94043, USA; Peter.Tenenbaum@nasa.gov, fergal.mullally@nasa.gov, jjenkins@mail.arc.nasa.gov, Douglas.A.Caldwell@nasa.gov

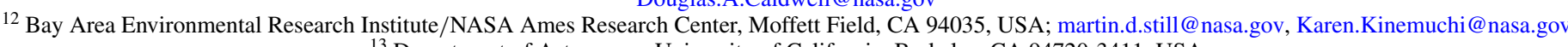

${ }^{13}$ Department of Astronomy, University of California, Berkeley, CA 94720-3411, USA

${ }^{14}$ Centre for Astrophysics Research, University of Hertfordshire, College Lane, Hatfield AL10 9AB, UK

15 Department of Astronomy, California Institute of Technology, Pasadena, CA 91109, USA; johnjohn@astro.caltech.edu

${ }^{16}$ Department of Physics, Southern Connecticut State University, New Haven, CT, 06515, USA; horche2@southernct.edu ${ }^{17}$ Space Telescope Science Institute, Baltimore, MD 21218, USA

18 Jet Propulsion Laboratory, California Institute of Technology, Pasadena, CA 91109, USA

${ }^{19}$ Department of Astronomy, University of Florida, Gainesville, FL 32611; eford@ astro.ufl.edu

${ }^{20}$ Department of Astronomy, Yale University, New Haven, CT 06510; debra.fischer@gmail.com

${ }^{21}$ National Optical Astronomy Observatory, Tucson, AZ 85719, USA

22 Solar System Exploration Division, NASA Goddard Space Flight Center, Greenbelt, MD 20771, USA

${ }^{23}$ Niels Bohr Institute, University of Copenhagen, DK-2100 Copenhagen, Denmark

${ }^{24}$ Centre for Star and Planet Formation, Natural History Museum of Denmark, University of Copenhagen, DK-1350 Copenhagen, Denmark ${ }^{25}$ Las Cumbres Observatory Global Telescope, Goleta, CA 93117, USA

${ }^{26}$ Department of Physics and Astronomy, San Jose State University, San Jose, CA, 95192, USA; natalie.m.batalha@nasa.gov Received 2011 July 8; accepted 2011 September 1; published 2011 October 11

\begin{abstract}
We report the detection of three transiting planets around a Sun-like star, which we designate Kepler-18. The transit signals were detected in photometric data from the Kepler satellite, and were confirmed to arise from planets using a combination of large transit-timing variations (TTVs), radial velocity variations, Warm-Spitzer observations, and statistical analysis of false-positive probabilities. The Kepler- 18 star has a mass of $0.97 M_{\odot}$, a radius of $1.1 R_{\odot}$, an effective temperature of $5345 \mathrm{~K}$, and an iron abundance of $[\mathrm{Fe} / \mathrm{H}]=+0.19$. The planets have orbital periods of approximately 3.5, 7.6, and 14.9 days. The innermost planet "b" is a "super-Earth" with a mass of $6.9 \pm 3.4 M_{\oplus}$, a radius of $2.00 \pm 0.10 R_{\oplus}$, and a mean density of $4.9 \pm 2.4 \mathrm{~g} \mathrm{~cm}^{3}$. The two outer planets "c" and "d" are both low-density Neptune-mass planets. Kepler-18c has a mass of $17.3 \pm 1.9 M_{\oplus}$, a radius of $5.49 \pm 0.26 R_{\oplus}$, and a mean density of $0.59 \pm 0.07 \mathrm{~g} \mathrm{~cm}^{3}$, while Kepler-18d has a mass of $16.4 \pm 1.4 M_{\oplus}$, a radius of $6.98 \pm 0.33 R_{\oplus}$ and a mean density of $0.27 \pm 0.03 \mathrm{~g} \mathrm{~cm}^{3}$. Kepler-18c and Kepler-18d have orbital periods near a 2:1 mean-motion resonance, leading to large and readily detected TTVs.
\end{abstract}

Key words: planetary systems - stars: individual (Kepler-18, KIC 8644288, 2MASS J19521906+4444467) techniques: photometric - techniques: spectroscopic

Online-only material: color figures

\section{INTRODUCTION}

\footnotetext{
* Based in part on observations obtained at the W. M. Keck Observatory, which is operated by the University of California and the California Institute of Technology.
}

Kepler is a NASA mission designed to detect the transits of exoplanets across the disks of their stars. The mission's ultimate goal is to detect the transits of potentially habitable Earth-size 
planets. To achieve this goal requires a telescope in a very stable space environment with a large $(0.95 \mathrm{~m})$ effective aperture monitoring the brightness of about 150,000 stars simultaneously and continuously for over three years. The Kepler Mission design and performance are summarized by Borucki et al. (2010a) and by Koch et al. (2010a), and a discussion of the commissioning and first quarter data are given by Borucki et al. (2011a). Borucki et al. (2011b) reported 1235 planet candidates that were discovered during the first four months of the mission. Batalha et al. (2010a) discuss the selection and characteristics of the Kepler target stars.

The first five planets discovered by the Kepler mission (Kepler 4-8) were reported in 2010 January (Borucki et al. 2010b; Koch et al. 2010b; Dunham et al. 2010; Latham et al. 2010; Jenkins et al. 2010a). Kepler has detected an abundance of multi-planet systems. Borucki et al. (2011b) reported a total of 170 candidate multi-planet systems among the 997 planet candidate host stars from the 2011 February data release. Steffen et al. (2010) presented five of these systems in detail. The Kepler-9b and c system (Holman et al. 2010) was the first transiting multi-planet system confirmed by transit-timing variations (TTVs). Kepler-10 (Batalha et al. 2011) was the first rocky planet found by Kepler. Kepler-11 (Lissauer et al. 2011a) is a transiting system of six planets.

Not all Kepler planets can be directly confirmed by supporting reflex radial velocity (RV) measurements of the parent star, or by detection and modeling of TTVs. Instead, some planets must be validated by analyzing all possible astrophysical false-positive scenarios and comparing their a priori likelihood to that of a planet. The Kepler project has been able to utilize the BLENDER technique developed by Torres et al. (2004) to validate a third planet in the Kepler-9 system (Torres et al. 2011), a second planet in the Kepler-10 system (Fressin et al. 2011), and the outer planet in the Kepler-11 system (Lissauer et al. 2011a).

Here, we present the Kepler-18 system, containing two Neptune-mass transiting planets near a 2:1 mean-motion resonance, which show significant gravitational interactions that are observed via measurements of TTVs, as well as a small, inner super-Earth-size transiting planet. This system is remarkably similar to the Kepler-9 system in its overall architecture.

\section{Kepler PHOTOMETRY}

The Kepler spacecraft carries a photometer with a widefield $\left(\sim 115 \mathrm{deg}^{2}\right)$ Schmidt camera of $0.95 \mathrm{~m}$ effective aperture. The spacecraft was launched in 2009 March, and is now in an Earth-trailing heliocentric orbit which allows nearly continuous photometric coverage of its field of view in Cygnus and Lyra. Caldwell et al. (2010) discuss the early instrumental performance of the Kepler photometer system. The primary data for detection of transiting planets are the Long Cadence (LC) "Pre-search Data Conditioned" (PDC) time series data, in which 270 consecutive CCD readouts are binned, giving an effective sampling interval of 29.4244 minutes (Jenkins et al. 2010b). A small selected subset of Kepler targets is sampled at the Short Cadence (SC) rate of nine consecutive reads for a sampling interval of $58.85 \mathrm{~s}$ (Gilliland et al. 2010). Thus, one LC sample is the sum of $30 \mathrm{SC}$ samples. The data from the spacecraft are processed through the Kepler Science Operations Center pipeline (Jenkins et al. 2010c) to perform standard CCD processing and to remove instrumental artifacts. The LC PDC time series data are searched for possible planetary transits using a wavelet-based adaptive matched filter (Jenkins et al. 2010d). Possible planetary transit events with amplitude greater than
$7.1 \sigma$ are flagged and are then subjected to intensive validation efforts using the Kepler data (Batalha et al. 2010b; Wu et al. 2010). Objects that pass this level of vetting are designated as a "Kepler Object of Interest" (KOI) and are sent to the Follow-up Observing Program (FOP) for further study.

\subsection{Light Curves and Data Validation}

One of the objects identified with possible transiting planets is the $K p 13.549 \mathrm{mag}$ (where $K p$ is the magnitude in the Kepler passband) star KIC 8644288 (2MASS J19521906+4444467, K00137). After a possible transiting planet has been detected, the Kepler data are subjected to a set of statistical tests to search for possible astrophysical false-positive origin of the observed signal. These data validation tests for the first five Kepler planet discoveries are described by Batalha et al. (2010b). Additional tests, including measurement of the image centroid motion during a transit (Wu et al. 2010) all gave a high probability that the signals seen were real. The application of these techniques to Kepler-10b is described in detail by Batalha et al. (2011).

Two separate transiting objects were immediately obvious in the LC data. K00137.01 has a transit ephemeris of $T_{0}$ [BJD] $=$ $(2455167.0883 \pm 0.0023)+N *(7.64159 \pm 0.00003)$ days and a transit depth of $2287 \pm 9 \mathrm{ppm}$. (All transit times and ephemerides in this paper are based on UTC.) K00137.02 has an ephemeris of $T_{0}$ [BJD] $=(2455169.1776 \pm 0.0013)+N *(14.85888 \pm$ $0.00004)$ days and a transit depth of $3265 \pm 12 \mathrm{ppm}$. It was noted that the orbits of these two objects were very near a period ratio of 2:1. After filtering these transits of K00137.01 and K00137.02 from the light curve, we searched again for transiting objects and found a third planet candidate in the system, K00137.03, which has a shorter orbital period than the other two transiting planets. K00137.03 has the ephemeris $T_{0}[\mathrm{BJD}]=$ $(2454966.5068 \pm 0.0021)+N *(3.504725 \pm 0.000028)$ days, and a transit depth of only $254 \pm 8 \mathrm{ppm}$. The light curve for K00137 is shown in Figure 1. The upper panel shows the raw uncorrected "photometric analysis" (PA) light curves that come out of the data processing pipeline, and the lower panel shows the corrected PDC light curves. The two transit events that look significantly deeper than the others, near BJD 2454976.0 and BJD 2455243.5 are simultaneous transits of K00137.01 and K00137.02.

The folded light curves for each of the three candidate planets are shown in Figure 2. This figure shows the light curves folded on the ephemeris given above for each KOI. The significant width of the ingress and egress for Kepler-18c and Kepler-18d is a result of the transit time variations discussed in Section 6.

\section{FOLLOW-UP OBSERVATIONS}

After the possible transiting planets were found, and the KOIs passed the Data Validation tests for false-positive signals, K00137 was sent on to the Kepler FOP for ground-based telescopic observations designed either to find any additional indication that these KOIs might be an astrophysical falsepositive signal, or to verify the planetary nature of the transit events.

\subsection{Reconnaissance Spectroscopy}

The first FOP step is to obtain high spectral resolution, low signal-to-noise ratio $(\mathrm{S} / \mathrm{N})$ spectroscopy in order to verify the Kepler Input Catalog (KIC) stellar classification, and to search for evidence of stellar multiplicity in the spectrum. Spectra from the Hamilton echelle spectrograph on the Lick Observatory $3 \mathrm{~m}$ 


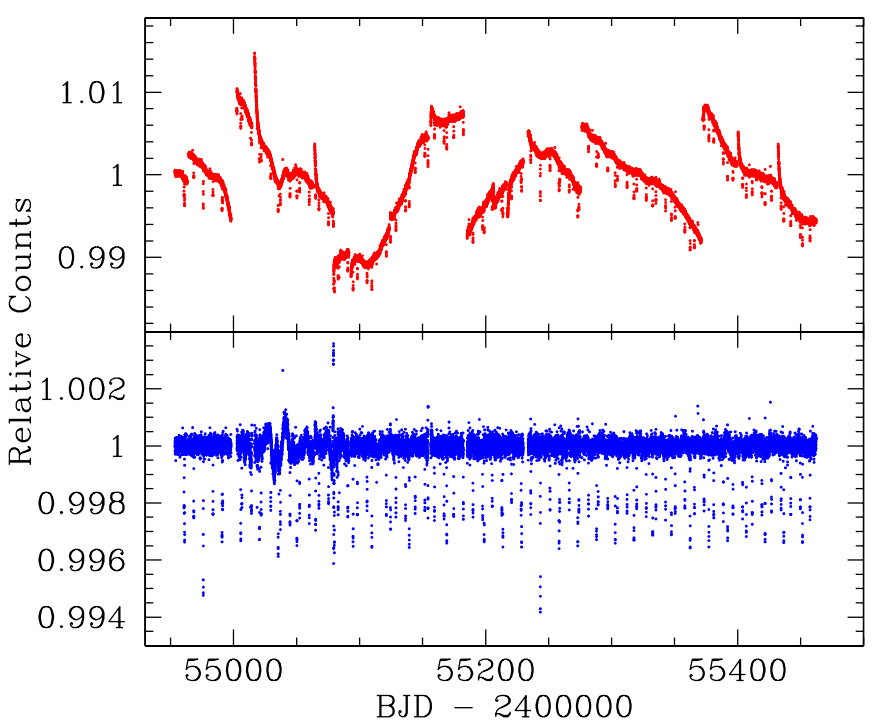

Figure 1. Kepler light curves for K00137. The upper panel shows the normalized raw PA light curves for quarters 0 through 6 . The various discontinuities are due to effects such as spacecraft safe-mode events or loss of fine pointing. Each quarter put the star on a different detector, which accounts for the change in overall sensitivity. The long-term drifts in each quarter are temperature related. The lower panel shows the normalized corrected PDC light curve. Most of the spacecraft-related variability of the PA light curve has been removed.

(A color version of this figure is available in the online journal.)

Shane Telescope were obtained on the nights of 2009 August 8 and 9, and on 2009 September 1 UT. These spectra showed no convincing evidence for RV variability at the $0.5 \mathrm{~km} \mathrm{~s}^{-1}$ level, and no hints of any contaminating spectra. These spectra were cross-correlated against a library of synthetic stellar spectra as described by Batalha et al. (2011), in order to derive basic stellar parameters to compare with the KIC values. These spectra yielded $T_{\text {eff }}=5250 \pm 125 \mathrm{~K}, \log g=4.0 \pm 0.25$, and $V \sin i=2 \pm 2 \mathrm{~km} \mathrm{~s}^{-1}$. The height of the cross-correlation peaks ranged from 0.82 to 0.89 , indicating a very good match with the library spectra.

\subsection{High Spatial Resolution Imaging}

High-resolution imaging of the surroundings of a KOI is an important step to identify possible sources of false-positive signals. We need to ensure that the detected transit signal is indeed originating on the selected target star, and not on a background star that was unresolved in the original KIC imaging of the Kepler field.

A seeing limited image was obtained at Lick Observatory's $1 \mathrm{~m}$ Nickel telescope using the Direct Imaging Camera. This image was a single one-minute exposure taken in the $I$ band, with seeing of approximately 1 1.5. The only nearby star is a faint object (approximately 5 mag fainter than K00137) about 5 '.5 to the north. A $J$-band image of the $1^{\prime} \times 1^{\prime}$ field of view around $\mathrm{K} 00137$ was taken as part of a complete $J$-band survey of the Kepler field of view using the wide-field camera on the United Kingdom Infrared Telescope. These images have a typical spatial resolution of $0.8-0$ '.9 and an image depth of $J=19.6$ (Vega system). This image also shows only a faint source about $5^{\prime \prime} .5$ to the north.

We perform speckle observations at the WIYN $3.5 \mathrm{~m}$ telescope, using the Differential Speckle Survey Instrument (DSSI; Horch et al. 2011; Howell et al. 2011). DSSI provides simultaneous observations in two filters by employing a dichroic beam

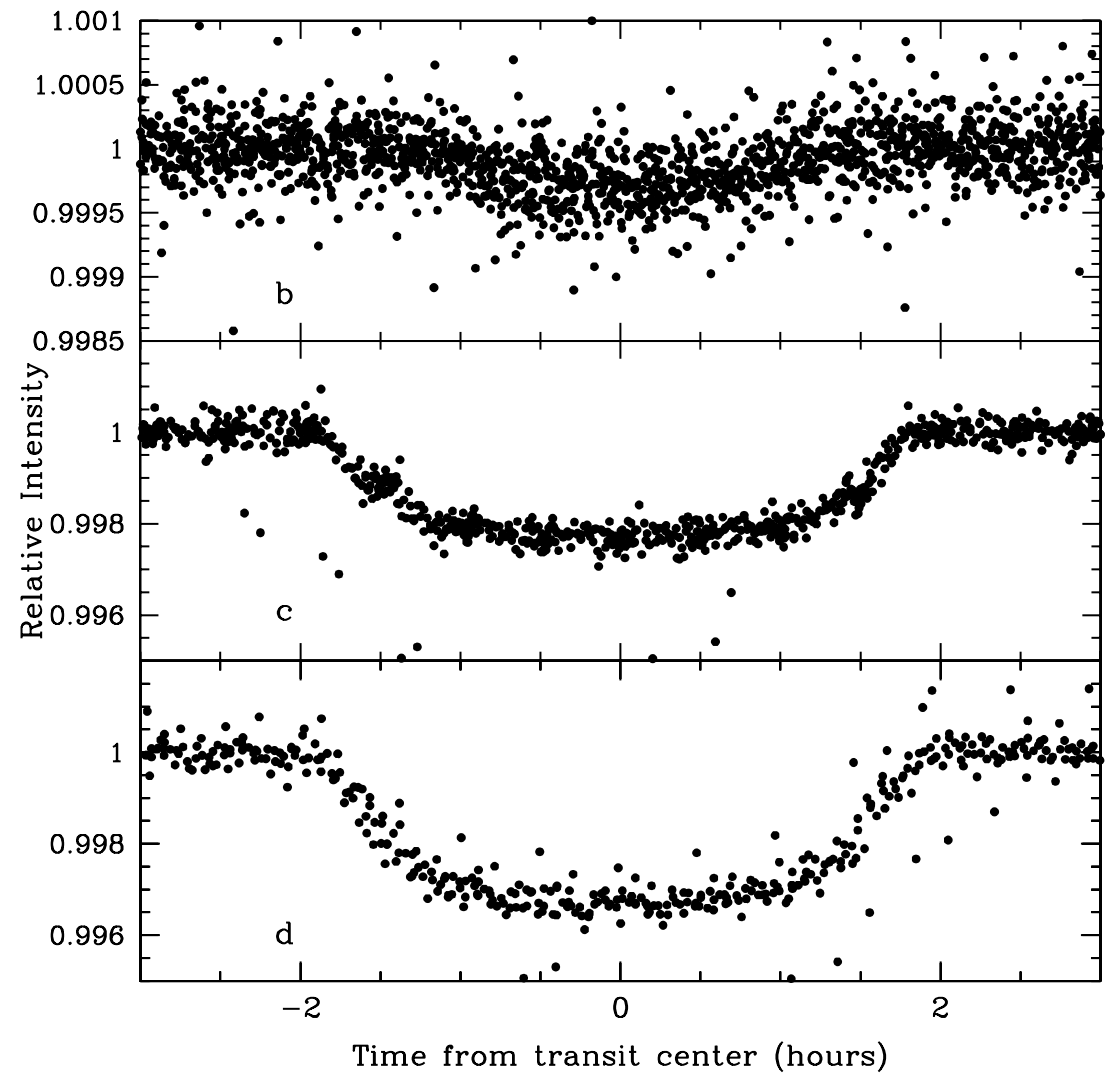

Figure 2. Folded light curves for K00137. The top row is Kepler-18b (K00137.03), the middle row is Kepler-18c (K00137.01), and the bottom row is Kepler-18d (K00137.02). The light curves are folded on the mean period listed in Table 4. 


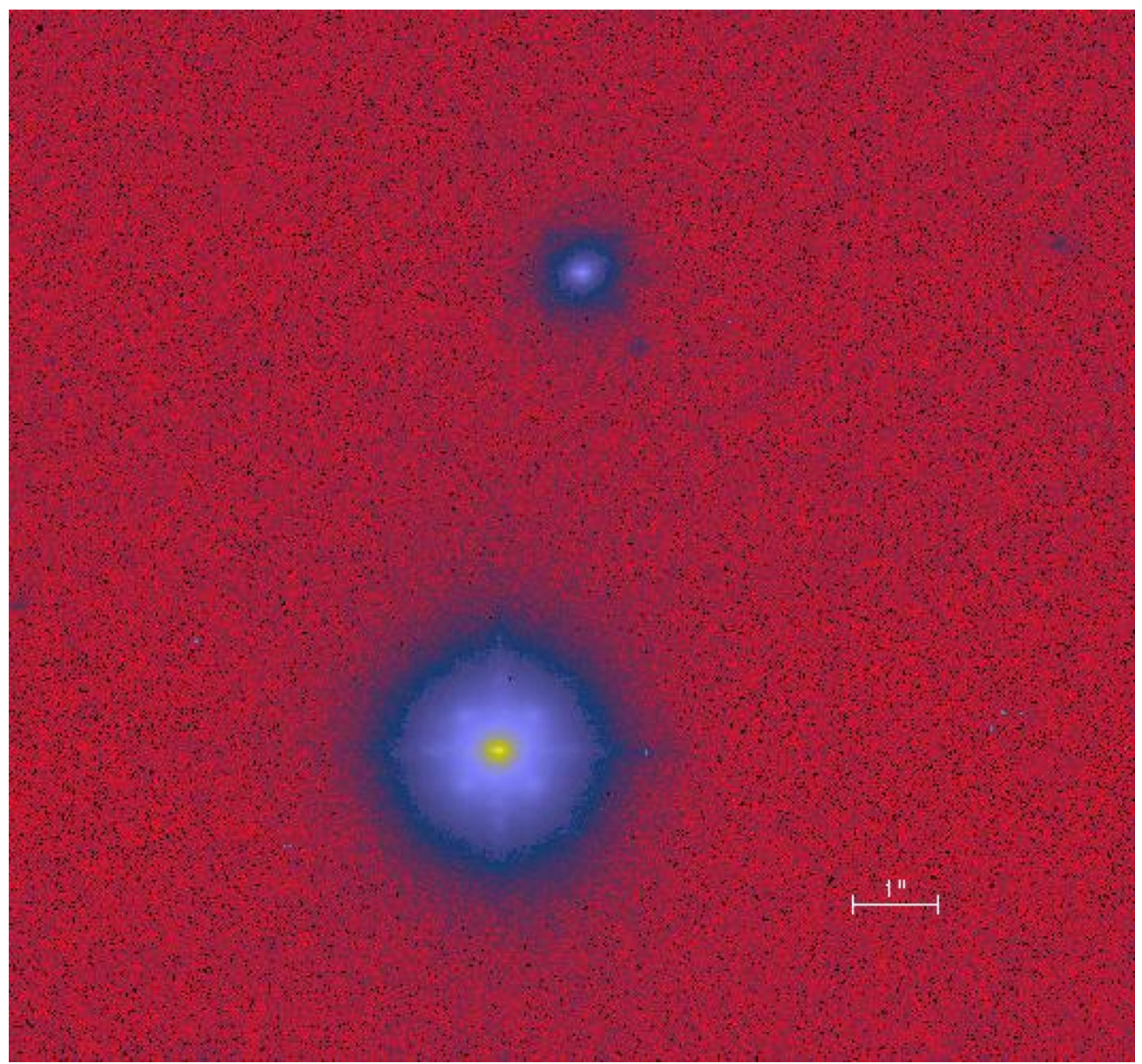

Figure 3. High spatial resolution A/O image of K00137 taken with the Palomar Observatory $5 \mathrm{~m}$ adaptive optics system. The nearest contaminating source is about $5 " .5$ to the north.

(A color version of this figure is available in the online journal.)

splitter and two identical EMCCDs as the imagers. We generally observe simultaneously in $V$ and $R$ bandpasses, where $V$ has a central wavelength of $5620 \AA$, and $R$ has a central wavelength of $6920 \AA$, and each filter has an FWHM of $400 \AA$. The speckle observations of K00137 were obtained on 2010 June 22 UT and consisted of three sets of 1000, $80 \mathrm{~ms}$ individual speckle images. Along with a nearly identical $V$-band reconstructed image, the speckle results reveal no companion star near K00137 within the annulus from 0 '. 05 to $1^{\prime \prime} .8$ to a limit $(5 \sigma)$ of 4.2 mag fainter in both $V$ and $R$ than the $K p 13.549$ target star.

Near-infrared adaptive optics (AO) imaging of K00137 was obtained on the night of 2009 September 8 UT with the Palomar Hale $5 \mathrm{~m}$ telescope and the Palomar High Angular Resolution Observer (PHARO) near-infrared camera (Hayward et al. 2001) behind the Palomar AO system (Troy et al. 2000). PHARO, a $1024 \times 20124 \mathrm{HgCdTe}$ infrared array, was utilized in 25.1 mas pixel ${ }^{-1}$ mode, yielding a field of view of $25^{\prime \prime}$. Observations were performed in the $J$ filter. The data were collected in a standard five-point quincunx dither pattern of $5^{\prime \prime}$ steps interlaced with an off-source (60" east) sky dither pattern. Data were taken with integration times per frame of $30 \mathrm{~s}$ with a total on-source integration time of 7.5 minutes. The $\mathrm{AO}$ system guided on the primary target itself and produced a central core width of FWHM $=0^{\prime} \cdot 075$. Figure 3 shows this Palomar image of K00137. There are two additional sources within 15" of the primary target. The first source, located 5".6 to the north of K00137, is 4 mag fainter at $J$; the second, located $15^{\prime \prime} 0$ to the southeast, is 5.8 mag fainter. To produce the observed transit depths in the blended Kepler photometry, the $\delta_{J}=4$ mag star would need to have eclipses that are 0.09 and 0.5 mag deep, and the $\delta_{J}=5.8 \mathrm{mag}$ star would need to have eclipses that are 0.15 and $1.3 \mathrm{mag}$ deep. Such deep eclipses from stars separated by more than 4" (>1 Kepler pixel) would easily be detected in the centroid motion analysis, but no centroid motion was detected between in and out of transit, indicating that these two stars were not responsible for the observed events. Six additional sources were detected at $J$ within $15^{\prime \prime}$ of the primary target. But all of these sources are $\delta_{J}>8$ mag fainter than K00137 and could not produce the observed transit events (i.e., even if the stars dimmed by $100 \%$, the resulting transit in the blended photometry would not be deep enough to match the observed transit depths).

Source detection completeness was evaluated by randomly inserted fake sources of various magnitudes in steps of $0.5 \mathrm{mag}$ and at varying distances in steps of 1.0 FWHM from the primary target. Identification of sources was performed both automatically with the IDL version of DAOPhot and by eye. Magnitude detection limits were set when a source was not detected by the automated FIND routine or was not detected by eye. Within a distance of 1-2 FWHM, the automated finding routine often failed even though the eye could discern two sources, but beyond that distance the two methods agreed well. A summary of the detection efficiency as a function of distance from the primary star is given in Table 1 . 
Table 1

Palomar AO Source Sensitivity as a Function of Distance from the Primary Target at $J$

\begin{tabular}{cccc}
\hline \hline $\begin{array}{c}\text { Distance } \\
(\text { FWHM })\end{array}$ & $\begin{array}{c}\text { Distance } \\
\left({ }^{\prime \prime}\right)\end{array}$ & $\begin{array}{c}\Delta J \\
(\mathrm{mag})\end{array}$ & $\begin{array}{c}J \\
(\mathrm{mag})\end{array}$ \\
\hline 1 & 0.075 & 1.5 & 13.7 \\
2 & 0.150 & 2.5 & 14.7 \\
3 & 0.225 & 3.5 & 15.7 \\
4 & 0.300 & 4.0 & 16.2 \\
5 & 0.375 & 4.5 & 16.7 \\
6 & 0.450 & 5.0 & 17.2 \\
7 & 0.525 & 6.0 & 18.2 \\
8 & 0.600 & 7.0 & 19.2 \\
9 & 0.675 & 7.5 & 19.7 \\
40 & 3.000 & 8.5 & 20.7 \\
\hline
\end{tabular}

Table 2

Mean Pixel Response Function Fit Source Offsets

\begin{tabular}{lcc}
\hline \hline Planet & $\begin{array}{c}\text { Distance } \\
\left({ }^{\prime \prime}\right)\end{array}$ & $\sigma$ \\
\hline Kepler-18b & $0.536 \pm 0.245$ & 2.19 \\
Kepler-18c & $0.070 \pm 0.107$ & 0.66 \\
Kepler-18d & $0.067 \pm 0.103$ & 0.65 \\
\hline
\end{tabular}

A major source of false-positive planet indication in the Kepler data is background eclipsing binary stars within the photometric aperture of Kepler-18, which, when diluted by Kepler-18 itself, can produce a planetary-size transit signal. We perform a direct measurement of the source location via difference images. Difference image analysis takes the difference between average in-transit pixel images and average out-oftransit images. Barring pixel-level systematics, the pixels with the highest flux in the difference image will form a star image at the location of the transiting object, with an amplitude equal to the depth of the transit. Performing a fit of the Kepler pixel response function (PRF; Bryson et al. 2010) to both the difference and out-of-transit images quantifies the offset of the transit source from Kepler-18. Difference image analysis is vulnerable to various systematics due to crowding and PRF errors which will bias the result (Bryson et al. 2010). These types of biases will vary from quarter to quarter. We ameliorate these biases by computing the uncertainty-weighted robust average of the source locations over available quarters. Table 2 gives the offsets of the transit signal source from Kepler-18 averaged over quarters 1 through 8 for all three-planet candidates. We see that the average offsets are within $1 \sigma$ of Kepler-18, with Kepler- $18 \mathrm{~b}$ being just over $2 \sigma$. From all of these lines of evidence, we conclude that there are no other objects within 4 mag near K00137 from 0.05 out to $15^{\prime \prime}$.

\subsection{Precise Doppler Measurements of Kepler-18}

After completion of the reconnaissance spectroscopy and high-resolution imaging, K00137 showed no evidence that might refute the planetary nature of the transit event in the Kepler light curve and the target was approved for high precision RV observations. We obtained 14 relative velocities with the Keck I High Resolution Echelle Spectrometer (HIRES; Vogt et al. 1994) between 2009 September 1 and 2010 August 28 UT. We used the same spectrograph configuration as is normally used for precise Doppler measurements of solar-type stars (cf. Marcy et al. 2008; Cochran et al. 2002). Decker B5 (0!.87 × 3".5) was used for the first five RV observations taken in 2009, and decker
Table 3

Keck HIRES Relative Radial Velocity Measurements of K00137

\begin{tabular}{lrc}
\hline \hline BJD & $\begin{array}{c}\mathrm{RV} \\
\left(\mathrm{m} \mathrm{s}^{-1}\right)\end{array}$ & $\begin{array}{c}\sigma \\
\left(\mathrm{m} \mathrm{s}^{-1}\right)\end{array}$ \\
\hline 2455076.008719 & 4.67 & 5.02 \\
2455076.927037 & 4.24 & 4.99 \\
2455081.024475 & 5.43 & 8.10 \\
2455082.007257 & 1.09 & 4.70 \\
2455084.983537 & -8.83 & 5.61 \\
2455318.066020 & 1.65 & 5.36 \\
2455322.029214 & -11.97 & 4.64 \\
2455373.003937 & 10.85 & 4.52 \\
2455403.018946 & 21.47 & 5.85 \\
2455405.909151 & -12.24 & 4.73 \\
2455406.881186 & -0.53 & 4.39 \\
2455413.010870 & -10.44 & 5.24 \\
2455432.969613 & 1.21 & 4.45 \\
2455436.781954 & -8.54 & 4.45 \\
\hline
\end{tabular}

$\mathrm{C} 2\left(0^{\prime} .87 \times 14^{\prime \prime}\right)$ was used for all of the later spectra. Exposures taken with the B5 decker entrance to the HIRES spectrometer suffer RV errors of up to $\pm 15 \mathrm{~m} \mathrm{~s}^{-1}$ when the moon is full and the relative Doppler shift of the star and solar spectrum is less than $10 \mathrm{~km} \mathrm{~s}^{-1}$ (i.e., within a line width). This $15 \mathrm{~m} \mathrm{~s}^{-1}$ error was determined from 10 stars for which observations were taken with and without moonlight subtraction. The first five RV measurements of Kepler-18 were made with the B5 decker and hence suffer from such errors. Decker C2 is long enough to permit us to do accurate subtraction of any moonlit sky spectrum. Sky subtraction was not possible with the spectra taken with decker B5. The iodine absorption cell was used as the velocity metric. This HIRES configuration can give a velocity precision as good as $1.0 \mathrm{~m} \mathrm{~s}^{-1}$, depending on the stellar spectral type, the stellar rotation velocity $V \sin i$, and on the $\mathrm{S} / \mathrm{N}$ of the observation. The exposure times for the K00137 spectra ranged from 2300 to $2700 \mathrm{~s}$, and the final S/Ns ranged from 63 to 80 pixel $^{-1}$, or 127 to 162 per 4.1 pixel resolution element. The Doppler RV analysis algorithm (Butler et al. 1996; Johnson et al. 2009) computes an uncertainty in each data point from the variance about the mean of the individual spectral chunks into which the spectrum is divided. Main-sequence stars having $T_{\text {eff }}$ near $5400 \mathrm{~K}$ have been measured for precise RVs for roughly 100 stars using the same HIRES instrument. Such stars typically show an additional noise of $2 \mathrm{~m} \mathrm{~s}^{-1}$, caused by surface velocity fields and instrumental effects. We have modeled this additional intrinsic short-term stellar variability by adding that $2.0 \mathrm{~m} \mathrm{~s}^{-1}$ "jitter" in quadrature to the uncertainties of each RV data point computed by the RV code. The measured HIRES relative velocities are given in Table 3 . These velocity measurements are shown as the black points in Figure 4. The solid blue line in this figure is the model fit from the joint Markov Chain Monte Carlo (MCMC) solution of the RV data and the light curve, presented in Section 4. The rms scatter of the RV observations around this model fit is $4.3 \mathrm{~m} \mathrm{~s}^{-1}$, whereas the rms scatter of the raw RVs is $9.6 \mathrm{~m} \mathrm{~s}^{-1}$. The inferred planetary masses from this solution are given in the first row of Table 8. Also shown as the dashed red line in Figure 4 is a two-planet RV solution that includes only Kepler-18c (K00137.01) and Kepler-18d (K00137.02). The black line in Figure 4 shows the velocities from the full multibody dynamical solution discussed in Section 6. The masses from this dynamical solution are adopted in Section 6 as our best determination of the planet masses. 

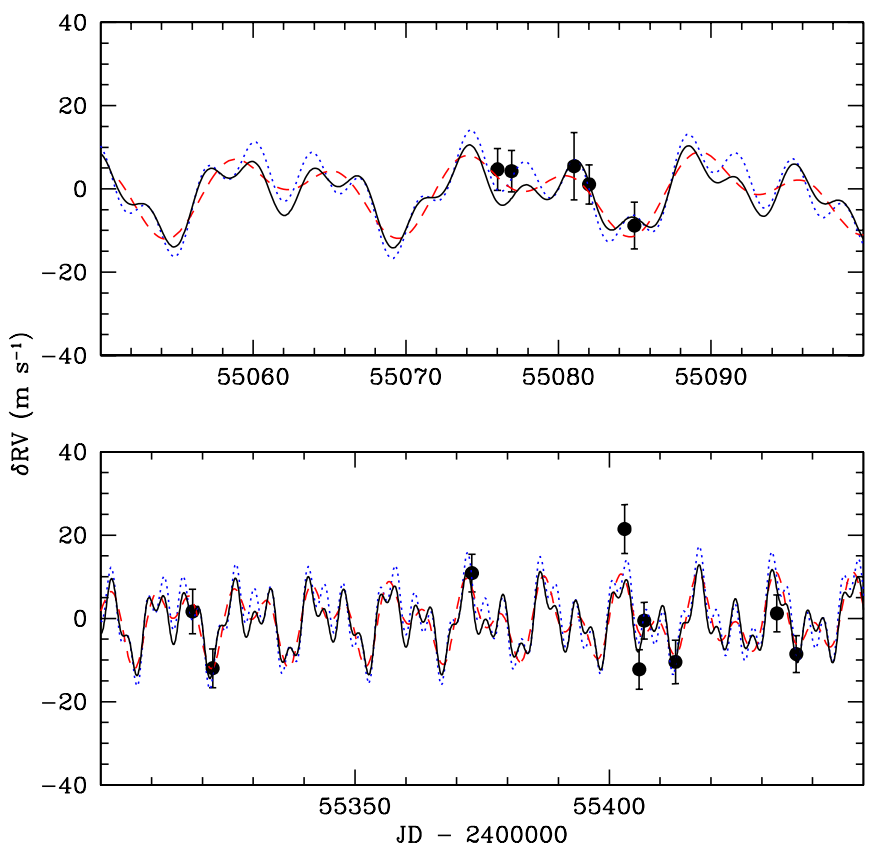

Figure 4. Observed precise relative radial velocity measurements from the Keck HIRES spectrograph. The solid black line gives the relative radial velocity variations of Kepler-18 computed from the full multi-body dynamical model discussed in Section 6. The dotted (blue) line is the model fit from the combined MCMC solution to the Kepler light curve and these velocity data presented in Section 4. A Keplerian fit from the radial velocity data alone, without the light curve constraints, is indistinguishable from this curve. The dashed (red) line is a two-planet Keplerian fit to the RV data accounting for Kepler-18c (K00137.01) and Kepler-18d (K00137.02) only.

(A color version of this figure is available in the online journal.)

\subsection{Spectroscopic Analysis}

We determined stellar parameters using the local thermodynamic equilibrium line analysis and spectral synthesis code MOOG $^{27}$ (Sneden 1973), together with a grid of Kurucz ${ }^{28}$ ATLAS9 model atmospheres. The method used is virtually identical to that described in Brugamyer et al. (2011). We analyzed a spectrum of Kepler-18 obtained with the Keck I HIRES spectrograph on 2010 August 27 UT. We first measured the equivalent widths of a carefully selected list of 53 neutral iron lines and 13 singly ionized iron lines in a spectrum of the Jovian satellite Ganymede, taken using the same instrumental setup and configuration as that used for Kepler-18. MOOG force-fits abundances to match these measured equivalent widths, using declared atomic line parameters. By assuming excitation equilibrium, we constrained the stellar temperature by eliminating any trends with excitation potential; assuming ionization equilibrium, we constrained the stellar surface gravity by forcing the derived iron abundance using neutral lines to match that of singly ionized lines. The microturbulent velocity $\xi$ was constrained by eliminating any trend with reduced equivalent width $\left(W_{\lambda} / \lambda\right)$. Our derived stellar parameters for the Sun (using our Ganymede spectrum) are as follows: $T_{\text {eff }}=5785 \pm 70 \mathrm{~K}, \log g=4.54 \pm 0.09 \mathrm{dex}$, microturbulent velocity $\xi=1.17 \pm 0.06 \mathrm{~km} \mathrm{~s}^{-1}$, and $\mathrm{Fe}$ abundance $\log (\epsilon)=7.54 \pm 0.05$ dex.

We repeated the process described above for the spectrum of Kepler-18. We then took the difference, on a line-by-line basis, of the derived iron abundance from each line. Our resulting iron

\footnotetext{
27 Available at http://www.as.utexas.edu/ chris/moog.html.

28 http://kurucz.harvard.edu/grids.html
}

abundance is therefore differential with respect to the Sun. To estimate the rotational velocity of Kepler-18, we synthesized three $5 \AA$ wide spectral regions in the range 5640-5690 and adjusted the Gaussian and rotational broadening parameters until the best fit (by eye) was found to the observed spectrum. The results of our analysis yield the following stellar parameters for Kepler-18: $T_{\text {eff }}=5345 \pm 100 \mathrm{~K}, \log g=4.31 \pm 0.12$, $\xi=1.09 \pm 0.08 \mathrm{~km} \mathrm{~s}^{-1},[\mathrm{Fe} / \mathrm{H}]=+0.19 \pm 0.06$, and $V \sin i<4 \mathrm{~km} \mathrm{~s}^{-1}$. The sky-projected rotational velocity of the star is very small. For such small $V \sin i$ values, disentangling line-broadening effects due to the instrument, macroturbulence, and rotation is difficult, at best, and requires higher signal to noise and resolution than our spectrum offers. In our MOOG analysis, we have therefore chosen to quote an upper limit for $V \sin i$, which we estimate by assuming that all broadening is due to stellar rotation.

A completely independent analysis of the same spectrum was done using the stellar spectral synthesis package SME (Valenti \& Piskunov 1996; Valenti \& Fischer 2005). This analysis of Kepler-18 gives $T_{\text {eff }}=5383 \pm 44 \mathrm{~K}, \log g=4.41 \pm 0.10$, $[\mathrm{Fe} / \mathrm{H}]=+0.20 \pm 0.04$, and $V \sin i=0.4 \pm 0.5 \mathrm{~km} \mathrm{~s}^{-1}$. The agreement between the MOOG and the SME analyses of this star is excellent. We have arbitrarily selected the MOOG parameters as our adopted values.

\section{LIGHT CURVE AND RADIAL VELOCITY SOLUTION}

We performed a joint solution of the photometric light curve and the RV data in order to derive the orbital parameters as well as the physical characteristics of the planet candidates, in the same manner as described in detail by Batalha et al. (2011) for Kepler-10b. The transit light curves were modeled using the analytic formulation of Mandel \& Agol (2002), and the radial velocities were fit with a Keplerian orbit. We model the mean density of the star, and for each of the three-planet candidates we model the planetary radius, the orbital period, $T_{0}$, the impact parameter, $b$, and the RV amplitude, $K$. The eccentricity was fixed to 0.0 . Model parameters were determined by minimizing the $\chi^{2}$ statistic using a Levenberg-Marquardt technique. To determine the best-fit model parameter distributions, we used a MCMC method that has been optimized for highly correlated parameters (Gregory 2011). Stellar parameters were determined in a separate MCMC analysis using the Yonsei-Yale isochrones (Yi et al. 2001, et seq.) with $T_{\text {eff }},[\mathrm{Fe} / \mathrm{H}]$, and fitted value of the mean-stellar density as constraints. Figure 5 shows the parameter distribution functions resulting from the MCMC analysis. Table 4 lists the adopted system parameters. In most cases, these parameters came from the MCMC analysis. As discussed in Section 6, significant TTVs were detected in Kepler-18c and Kepler-18d. These TTVs would have been absorbed into the MCMC periods and epochs and their uncertainties. Therefore, we have adopted the periods and epochs from the TTV analysis, and these are reported in Table 4. The uncertainty in the epoch is the median absolute deviation of the transit times from this ephemeris, and the uncertainty in the period is this quantity divided by the number of orbits between the first and last observed transits. Similarly, the dynamical system model presented in Section 6 derives our best and most reliable masses for the three planets. These masses from the dynamical model are reported in Table 4. The masses from the MCMC model are listed in Table 8. The TTV dynamical model places tight upper limits on the orbital eccentricity of planets "c" and "d," as shown in Table 7. These eccentricities are small enough that they would not significantly change the parameters in Table 4. 

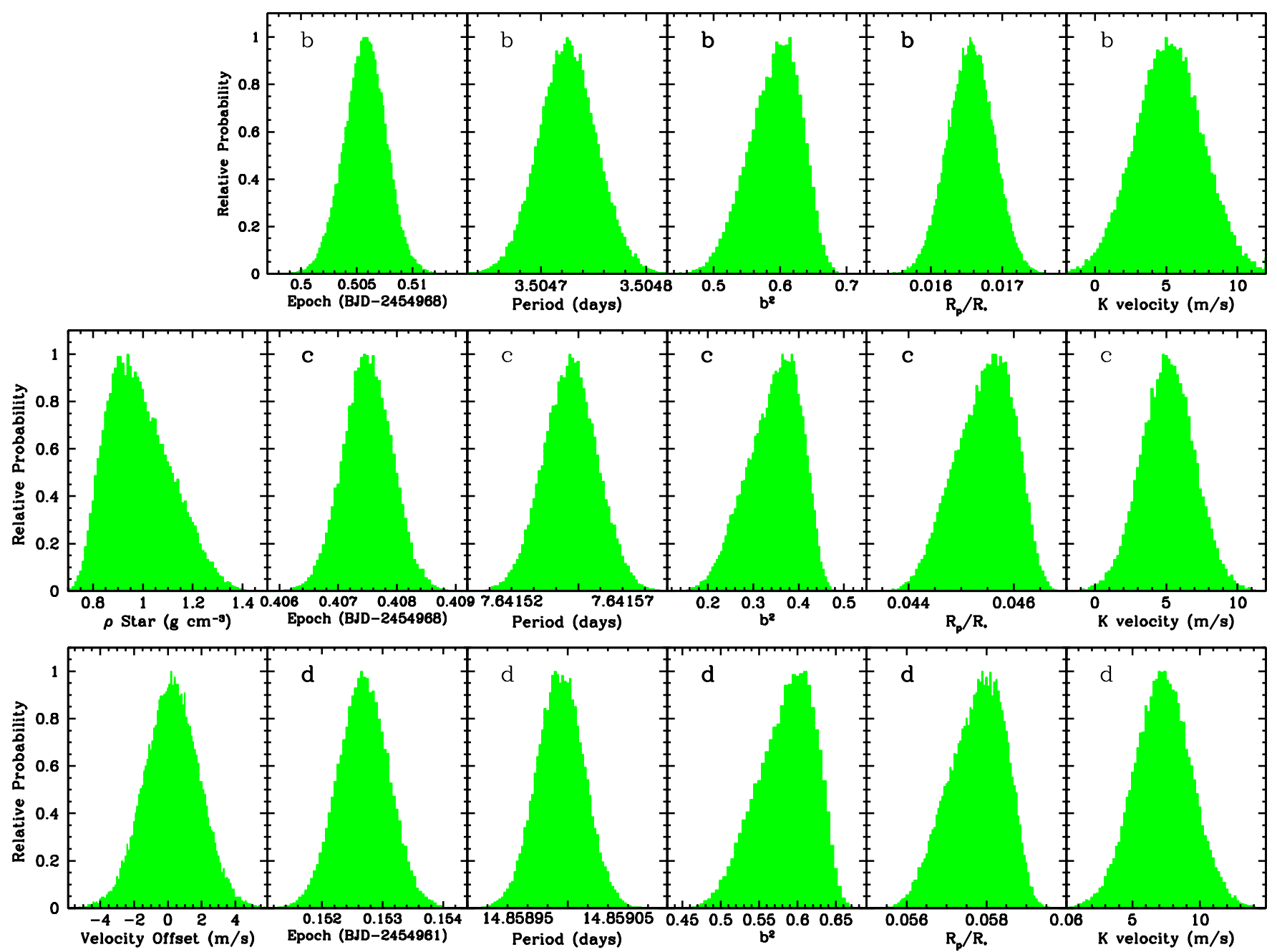

Figure 5. Markov Chain Monte Carlo model probability distribution functions for parameters of the Kepler-18 system. The left column gives the two stellar parameters of the stellar density and the relative radial velocity zero point. For the other five columns, the top row gives the planet parameters of transit epoch, orbital period, square of the impact parameter, ratio of the planetary to stellar radius, and the orbital velocity amplitude $K$ for Kepler-18b. The second and third rows give the same parameters for Kepler-18c and Kepler-18d, respectively.

(A color version of this figure is available in the online journal.)

For example, the zero eccentricity solution gives a stellar density of $1.01 \pm 0.12 \mathrm{~g} \mathrm{~cm}^{-3}$. Inclusion of the eccentricity from the TTV solution would change this by $0.03 \mathrm{~g} \mathrm{~cm}^{-3}$, or $0.25 \sigma$. For each parameter adopted from the MCMC model, we adopt the median value of the distribution, and the error bar represents the $68.3 \%$ confidence level—roughly equivalent to a $1 \sigma$ confidence level.

It is interesting and informative to compare the initial values for the transiting planet parameters presented in the tabulation of candidates by Borucki et al. (2011b) with those we have finally settled on in Table 4. The parameters of the parent star given by Borucki et al. (2011b) K00137 are simply taken from the KIC. The overall accuracy of the KIC photometric stellar parameters is actually quite good, as was shown by Brown et al. (2011). The other important planetary system parameters reported by Borucki et al. (2011b) are the orbital periods and transit depths of each of the three KOIs in the system. Our adopted orbital periods agree with the preliminary Borucki et al. (2011b) values to within $1 \sigma$ for K00137.01 and K00137.03, and within $4 \sigma$ for K00137.02. We note that Borucki et al. (2011b) assumed no transit time variations, and fit the best mean period to the data. The transit depths agree to within $2 \sigma$ for K00137.02 and K00137.03, but differ by 7.5 $\sigma$ for K00137.02. This slight disagreement for K00137.02 probably results from Borucki et al. (2011b) fitting an impact parameter of 0.03 , whereas our best value is $0.593 \pm 0.050$.

The RV variations of the parent star Kepler-18 resulting from this three-planet MCMC model is shown as the solid blue line in Figure 4. This solution is very similar to the three-body $\mathrm{RV}$ solution one would get from the RV data alone, holding the period and $T_{0}$ of each planet fixed at the values from the photometric light curve solution and assuming zero eccentricity. A two-body solution to the RV data considering only K00137.01 and K00137.02 is shown as the dashed red line in Figure 4. The full three-planet solution gives a significantly better fit to the observed radial velocities than does the two-body solution. The rms of the full MCMC three-body fit is $4.3 \mathrm{~m} \mathrm{~s}^{-1}$, while the rms of the two-body Keplerian solution is $5.5 \mathrm{~m} \mathrm{~s}^{-1}$. We computed an $f$-test based on the residuals of a two-planet and threeplanet RV fit. The periods where fixed based on transit data. We find $f=4.97$, which means there is a $0.68 \%$ probability that variance of the residuals is similar. Thus, this reduction in the rms supports the interpretation of K00137.03 as a third planetary companion to K00137. However, the RV observations 
Table 4

Adopted System Parameters

\begin{tabular}{lc}
\hline \hline Parameter & Value \\
\hline$M_{\star}\left(M_{\odot}\right)^{\mathrm{a}}$ & $0.972 \pm 0.042$ \\
$R_{\star}\left(R_{\odot}\right)^{\mathrm{a}}$ & $1.108 \pm 0.051$ \\
$\log L_{\star}\left(L_{\odot}\right)^{\mathrm{a}}$ & $-0.031 \pm 0.035$ \\
Age $(\mathrm{Gyr})^{\mathrm{a}}$ & $10.0 \pm 2.3$ \\
$\log g_{\star}{ }^{\mathrm{b}}$ & $4.31 \pm 0.12$ \\
$\rho_{\star}\left(\mathrm{g} \mathrm{cm}^{-3}\right)^{\mathrm{c}}$ & $1.01 \pm 0.12$ \\
\hline
\end{tabular}

\begin{tabular}{lc}
\hline \multicolumn{2}{c}{ Kepler-18b $=\mathrm{K} 00137.03$} \\
\hline$T_{0}{ }^{\mathrm{c}}$ & $2454966.5068 \pm 0.0021$ \\
$P(\text { days })^{\mathrm{c}}$ & $3.504725 \pm 0.000028$ \\
${\text { Transit depth }(\mathrm{ppm})^{\mathrm{c}}}_{b(\mathrm{impact} \text { parameter })^{\mathrm{c}}}$ & $254.0 \pm 7.8$ \\
$R_{p} / R_{\star}{ }^{\mathrm{c}}$ & $0.771 \pm 0.025$ \\
$M_{p}\left(M_{\oplus}\right)^{\mathrm{d}}$ & $0.01656 \pm 0.00032$ \\
$R_{p}\left(R_{\oplus}\right)^{\mathrm{c}}$ & $6.9 \pm 3.4$ \\
$i(\mathrm{deg})^{\mathrm{e}}$ & $2.00 \pm 0.10$ \\
$a / R_{\star} \mathrm{e}^{\mathrm{e}}$ & $84.92 \pm 0.26$ \\
$\left.\bar{\rho}_{p}(\mathrm{~g} \mathrm{~cm})^{-3}\right)^{3,4}$ & $8.58 \pm 0.37$ \\
$a(\mathrm{AU})^{\mathrm{e}}$ & $4.9 \pm 2.4$ \\
$\left.K(\mathrm{~m} \mathrm{~s})^{-1}\right)^{\mathrm{c}}$ & $0.0447 \pm 0.0006$ \\
$T_{14}(\mathrm{hr})^{\mathrm{e}}$ & $5.2 \pm 2.4$ \\
$T_{12}(\mathrm{hr})^{\mathrm{e}}$ & $2.076 \pm 0.036$ \\
\hline
\end{tabular}

\begin{tabular}{|c|c|}
\hline \multicolumn{2}{|c|}{ Kepler-18c $=$ K00137.01 } \\
\hline$T_{0}^{\mathrm{d}}$ & $2455167.0883 \pm 0.0023$ \\
\hline$P$ (days $^{\mathrm{d}}$ & $7.64159 \pm 0.00003$ \\
\hline Transit depth $(\mathrm{ppm})^{\mathrm{c}}$ & $2286.6 \pm 8.6$ \\
\hline$b$ impact parameter $)^{\mathrm{c}}$ & $0.593 \pm 0.050$ \\
\hline$R_{p} / R_{\star}{ }^{\mathrm{c}}$ & $0.04549 \pm 0.00055$ \\
\hline$M_{p}\left(M_{\oplus}\right)^{\mathrm{d}}$ & $17.3 \pm 1.9$ \\
\hline$R_{p}\left(R_{\oplus}\right)^{\mathrm{c}}$ & $5.49 \pm 0.26$ \\
\hline$i(\operatorname{deg})^{\mathrm{e}}$ & $87.68 \pm 0.22$ \\
\hline$a / R_{\star}^{\mathrm{e}}$ & $14.43 \pm 0.61$ \\
\hline $\bar{\rho}_{p}\left(\mathrm{~g} \mathrm{~cm}^{-3}\right)^{3,4}$ & $0.59 \pm 0.07$ \\
\hline$a(\mathrm{AU})^{\mathrm{e}}$ & $0.0752 \pm 0.0011$ \\
\hline$K\left(\mathrm{~m} \mathrm{~s}^{-1}\right)^{\mathrm{c}}$ & $5.1 \pm 1.9$ \\
\hline$T_{14}(\mathrm{hr})^{\mathrm{e}}$ & $3.488 \pm 0.020$ \\
\hline$T_{12}(\mathrm{hr})^{\mathrm{e}}$ & $0.229 \pm 0.022$ \\
\hline
\end{tabular}

\begin{tabular}{|c|c|}
\hline \multicolumn{2}{|c|}{ Kepler-18d = K00137.02 } \\
\hline$T_{0}^{\mathrm{d}}$ & $2455169.1776 \pm 0.0013$ \\
\hline$P$ (days) $^{\mathrm{d}}$ & $14.85888 \pm 0.00004$ \\
\hline Transit depth $(\mathrm{ppm})^{\mathrm{c}}$ & $3265 . \pm 12$ \\
\hline$b$ (impact parameter) $^{\mathrm{c}}$ & $0.767 \pm 0.024$ \\
\hline$R_{p} / R_{\star}{ }^{\mathrm{c}}$ & $0.05782 \pm 0.00069$ \\
\hline$M_{p}\left(M_{\oplus}\right)^{\mathrm{d}}$ & $16.4 \pm 1.4$ \\
\hline$R_{p}\left(R_{\oplus}\right)^{\mathrm{c}}$ & $6.98 \pm 0.33$ \\
\hline$i(\mathrm{deg})^{\mathrm{e}}$ & $88.07 \pm 0.10$ \\
\hline$a / R_{\star}{ }^{\mathrm{e}}$ & $22.48 \pm 0.96$ \\
\hline $\bar{\rho}_{p}\left(\mathrm{~g} \mathrm{~cm}^{-3}\right)^{3,4}$ & $0.27 \pm 0.03$ \\
\hline$a(\mathrm{AU})^{\mathrm{e}}$ & $0.1172 \pm 0.0017$ \\
\hline$K\left(\mathrm{~m} \mathrm{~s}^{-1}\right)^{\mathrm{c}}$ & $7.3 \pm 2.1$ \\
\hline$T_{14}(\mathrm{hr})^{\mathrm{e}}$ & $3.679 \pm 0.036$ \\
\hline$T_{12}(\mathrm{hr})^{\mathrm{e}}$ & $0.459 \pm 0.045$ \\
\hline
\end{tabular}

Notes.

${ }^{\text {a }}$ Based on isochrone fits using $\rho_{\star}$, from the MCMC model and $T_{\text {eff }}$ and $[\mathrm{Fe} / \mathrm{H}]$ from spectroscopy.

b From the MOOG analysis.

c From the MCMC model.

d From the TTV model.

e Derived from other parameters.

are not sufficiently dense to consider this to be a confirmation of this KOI as a planet.

\section{WARM-SPITZER OBSERVATIONS OF K00137.01 AND K00137.02}

Observation of the transits of the planets around Kepler-18 at multiple, widely spaced wavelengths is a valuable tool for confirming the planetary nature of the events. The depth of a planetary transit should be nearly independent of wavelength, aside from minor effects due to the possible finite brightness of the planet as a function of wavelength. In order to test for wavelength independence of the transit depths, K00137.01 and K00137.02 were observed during two transits with Warm-Spitzer/IRAC (Werner et al. 2004; Fazio et al. 2004) at $4.5 \mu \mathrm{m}$ (program ID 60028). The observations occurred on UT 2010 July 19 and UT 2010 August 13. Both visits lasted approximately $9 \mathrm{hr}$. The data were gathered in full-frame mode $(256 \times 256$ pixels $)$ with an exposure time of $12 \mathrm{~s}$ per image, which yielded 2418 and 2575 images per respective visit.

The method we used to produce photometric time series from the images is described by Désert et al. (2009). It consists of finding the centroid position of the stellar point-spread function (PSF) and performing aperture photometry using a circular aperture.

The images used are the Basic Calibrated Data delivered by the Spitzer archive. These files are corrected for dark current, flat-fielding, detector nonlinearity and converted into flux units. We convert the pixel intensities to electrons using the information given in the detector gain and exposure time provided in the FITS headers. This facilitates the evaluation of the photometric errors. We extract the UTC-based Julian date for each image from the FITS header (keyword DATE_OBS) and correct to mid-exposure. We convert to UTC-based BJD following the procedure developed by Eastman et al. (2010). We use the JPL Horizons ephemeris to estimate the position of the Spitzer Space Telescope during the observations. We correct for transient pixels in each individual image using a 20 point sliding median filter of the pixel intensity versus time. For this step, we compare each pixel's intensity to the median of the 10 preceding and 10 following exposures at the same pixel position and we replace outliers greater than $4 \sigma$ with their median value. The fraction of pixels we correct is lower than $0.16 \%$ for both transits. The centroid position of the stellar PSF is determined using DAOPHOT-type Photometry Procedures, GCNTRD, from the IDL Astronomy Library. ${ }^{29}$ We use the APER routine to perform aperture photometry with a circular aperture of variable radius, using radii of $1.5-8$ pixels, in 0.5 pixel steps. The propagated uncertainties are derived as a function of the aperture radius; we adopt the one which provides the smallest errors. We find that the transit depths and errors vary only weakly with the aperture radius for all the light curves analyzed in this project. The optimal aperture is found to be at 3.0 pixels. We estimate the background by fitting a Gaussian to the central region of the histogram of counts from the full array. The center of the Gaussian fit is adopted as the residual background intensity. As already seen in previous Warm-Spitzer observations (Deming et al. 2011; Beerer et al. 2011), we find that the background varies by $20 \%$ between three distinct levels from image to image, and displays a ramp-like behavior as a function of time. The contribution of the background to the total flux from the star is low for both observations, from $0.15 \%$ to $0.9 \%$ depending on the images. Therefore, photometric errors are not dominated by fluctuations in the background. We used a sliding median filter to select and trim outliers in flux and

\footnotetext{
29 http://idlastro.gsfc.nasa.gov/homepage.html
} 

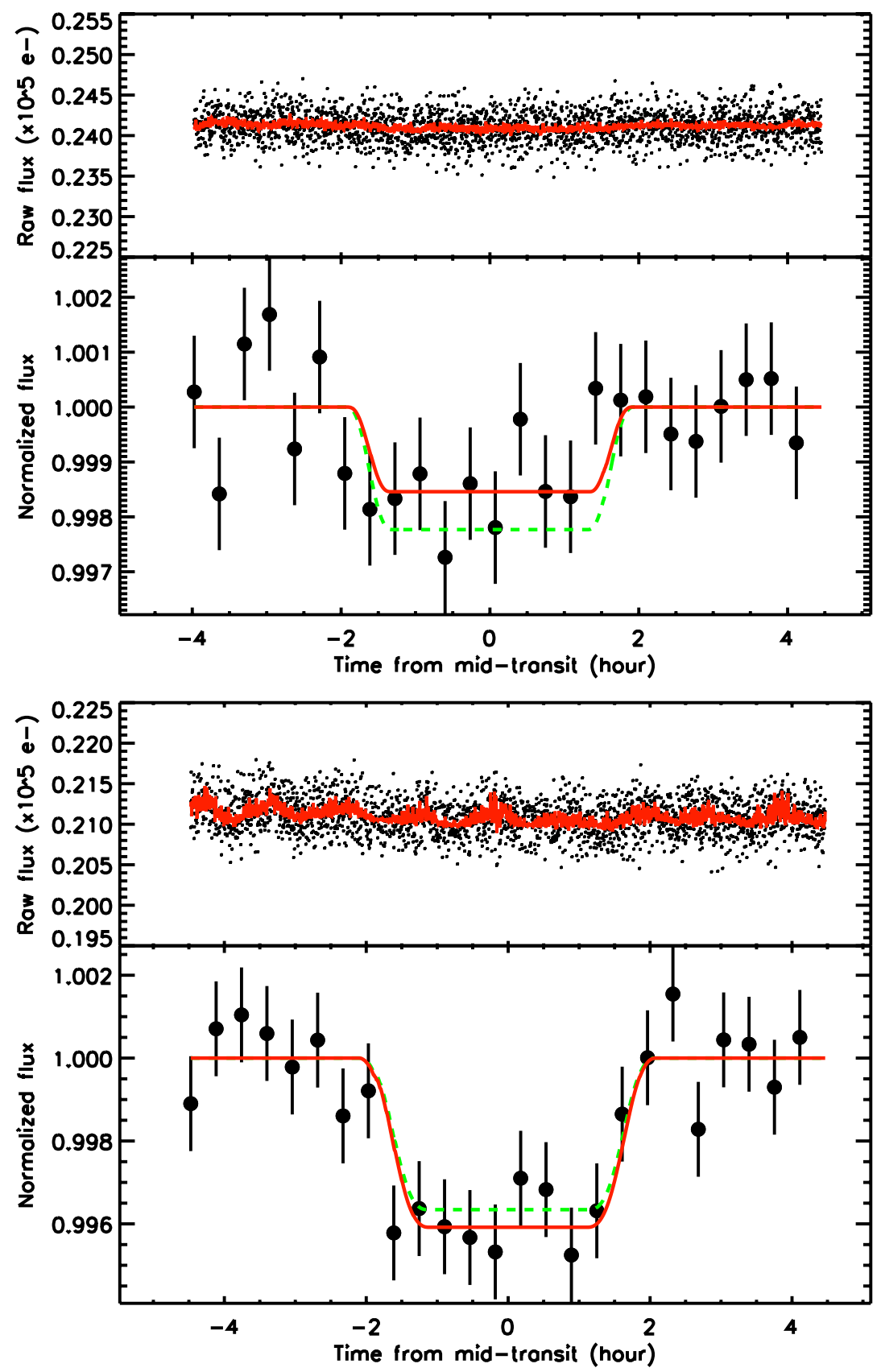

Figure 6. Spitzer transit light curves of K00137.01 (top) and K00137.02 (bottom) observed in the IRAC bandpass at $4.5 \mu \mathrm{m}$. Top panels: raw (unbinned) transit light curves. The red solid lines correspond to the best-fit models which include the time and position instrumental decorrelations as well as the model for the planetary transit (see details in Section 5). Lower panels: transit light curve corrected, normalized, and binned by 36 minutes. The best-fit Spitzer transit curves are plotted in red, and the transit shapes expected from the Kepler observations are overplotted in dashed green lines.

(A color version of this figure is available in the online journal.)

position greater than $5 \sigma$, which corresponds to $0.9 \%$ and $2.0 \%$ of the data, for the first and second visit, respectively. We also discarded the first half-hour of observations, which are affected by a significant telescope jitter before stabilization. The final number of photometric measurements used is 2246 and 2369. The raw time series are presented in the top panels of Figure 6. We find the typical $\mathrm{S} / \mathrm{N}$ to be 140 per image, which corresponds to $80 \%$ of the theoretical signal to noise. Therefore, the noise is dominated by Poisson photon noise.

\subsection{Analysis of the Warm-Spitzer Light Curves}

We used a transit light curve model multiplied by instrumental decorrelation functions to measure the transit parameters and their uncertainties from the Spitzer data as described in Désert et al. (2011b). We compute the transit light curves with the IDL transit routine OCCULTSMALL from Mandel \& Agol (2002). In the present case, this function depends on one parameter: the planet-to-star radius ratio $R_{p} / R_{\star}$. The orbital semimajor axis to stellar radius ratio (system scale) $a / R_{\star}$, the impact parameter $b$, and the time of mid-transit $T_{0}$ are fixed to the values derived from the Kepler light curves. The limb-darkening coefficients are set to zero since these Spitzer light curves do not have enough photometric precision to detect the curvature in the transit light curve that would be produced by limb darkening.

The Spitzer/IRAC photometry is known to be systematically affected by the so-called pixel-phase effect (see, e.g., 
Charbonneau et al. 2005; Knutson et al. 2008). This effect is seen as oscillations in the measured fluxes with a period of approximately 70 minutes (period of the telescope pointing jitter) and an amplitude of approximately $2 \%$ peak to peak. We decorrelated our signal in each channel using a linear function of time for the baseline (two parameters) and a quadratic function of the PSF position (four parameters) to correct the data for each channel. We checked that adding parameters to the correction function of the PSF position (as in Désert et al. 2009) does not improve the fit significantly. We performed a simultaneous Levenberg-Marquardt least-squares fit (Markwardt 2009) to the data to determine the transit and instrumental model parameters (seven in total). The errors on each photometric point were assumed to be identical and were set to the rms of the residuals of the initial best fit obtained. To obtain an estimate of the correlated and systematic errors (Pont et al. 2006) in our measurements, we use the residual permutation bootstrap, or "Prayer Bead," method as described in Désert et al. (2009). In this method, the residuals of the initial fit are shifted systematically and sequentially by one frame, and then added to the transit light curve model before fitting again. We allow asymmetric error bars spanning $34 \%$ of the points above and below the median of the distributions to derive the $1 \sigma$ uncertainties for each parameter as described in Désert et al. (2011a).

We measured the transit depths at $4.5 \mu \mathrm{m}$ of $1521_{-245}^{+277} \mathrm{ppm}$ for Kepler-18c and $4083_{-285}^{+298}$ for Kepler-18d. The values we measure for the transit depths in the Spitzer bandpass are in agreement at the $2 \sigma$ level compared to the Kepler bandpass. This indicates that the transit depths of Kepler-18c and Kepler$18 \mathrm{~d}$ are only weakly dependent on wavelength, if at all. This is in agreement with expectations for a dark planetary object, and indicates that there is no significant contamination from any nearby unresolved star of significantly different color. As discussed in Section 7, this is important evidence that the transit signals arise from planets and not from eclipsing stars blended with additional light.

These Spitzer observations provide a useful constraint on the kinds of false positives (blends) that may be mimicking the transit signal, such as eclipsing binaries blended with the target. If Kepler-18 were blended with an unresolved eclipsing binary of later spectral type that manages to reproduce the transit depth in the Kepler passband, the predicted depth at $4.5 \mu \mathrm{m}$ would be expected to be larger because of the higher flux of the contaminant at longer wavelengths compared to Kepler18. Since the transit depth we measure in the near-infrared is about the same as in the optical, this argues against blends composed of stars of much later spectral type. Based on model isochrones, the properties of the target star, and the transit depths measured with Spitzer at the $3 \sigma$ level, we determine a lower limit to the blend masses of 0.86 and $0.79 M_{\odot}$ for Kepler-18c and Kepler-18d, respectively.

\section{TRANSIT TIMING VARIATIONS ANALYSIS}

\subsection{Transit Times and Errors}

Kepler-18 was observed at the 29.4244 minute LC rate for the first three observing periods Q0-Q2. After the transits of K00137.01 and K00137.02 were detected, Kepler-18 was observed at the $58.85 \mathrm{~s} \mathrm{SC}$ in order to facilitate the measurement of possible TTVs. These SC data were used for Q3 through Q7. Transit times and their errors for each member of the Kepler-18 system were determined through an iterative procedure; a single step of this procedure is described as follows.
First, the detrended photometric data within four transit durations at each epoch were shifted by the current best-fit mid-transit times, and the light curves were folded on these transit times to form a transit template. This template was then fit with a transit light curve model (Mandel \& Agol 2002). Next, at each individual epoch, this light curve template was shifted in time and compared to the data by computing the standard $\chi^{2}$ statistic. This statistic was computed for a dense, uniform sample of mid-transit times centered on the current best-fit time and spanning approximately five current best-fit timing errors. The time $t_{0}$, corresponding to the minimum $\chi^{2}$, was recorded as the new best-fit mid-transit time. The $\chi^{2}$ data were then fit with a quadratic function of time, $\chi^{2}(t)=C\left(t-t_{0}\right)^{2}+\chi_{0}^{2}$. By choosing this functional form, we are assuming that the posterior likelihood is well described by a Gaussian function of the mid-transit time. The fidelity of the quadratic approximation was verified visually at each epoch. The timing error, $\sigma_{t}$, was found by solving for the time, $t=t_{0}+\sigma_{t}$, at which the quadratic model indicated a $\Delta \chi^{2}=1$. This corresponds to $\sigma_{t}=C^{-1 / 2}$.

This iterative procedure of template generation followed by timing estimate generally converged to the final values in two to three steps. The measured transit times for each observed transit of Kepler-18c are given in Table 5, and the measured transit times for Kepler-18d are given in Table 6.

A drift in the orbital inclination was measured for each planet by augmenting the nominal transit model with an epochdependent linear inclination (viz., $i(E)=i(0)+(\Delta i / \Delta E) \times E)$, and then fitting for the linear coefficient. In this fit, transit times were fixed to their best-fit values while the remaining transit parameters were allowed to vary. The best-fitting solution was found by minimizing the standard $\chi^{2}$ metric. The uncertainty was estimated by fitting a multivariate Gaussian to a sampling of the posterior parameter distribution. The drifts $(\Delta i / \Delta E)$ were found to be $[10 \pm 9,4 \pm 22,-3 \pm 10] \times 10^{-4}$ degrees per epoch for planets $b, c$, and $d$, respectively. Thus, we detect no secular inclination drift.

\subsection{Transit Time Variation Analysis}

To model the transit times and radial velocities, we applied the numerical routines that were previously used for Kepler-9 and -11 (Fabrycky 2010; Holman et al. 2010; Lissauer et al. 2011a). That is, a Levenberg-Marquardt $\chi^{2}$-minimization routine drove three-planet dynamical integrations, which calculated the transit times resulting from given orbital parameters. As in Lissauer et al. (2011a), we chose the parameters $\left(m_{p}, P, T_{0}, e \cos \omega\right.$, $e \sin \omega$ ) for each planet: mass, orbital period, transit phase, and eccentricity vector components. These parameters are osculating Jacobian orbital elements at the epoch 2455168.0 [BJD]. As in Holman et al. (2010), we also used the integrations to calculate radial velocities of the star at each of the observed times. We assumed $M_{\star}=0.95 M_{\odot}$, assumed the orbits are edge-on and coplanar, and neglected light-travel time effects in these numerical calculations.

The main data constraining the orbital model are the transit times of Table 5 ( 75 data points) and Table 6 (37 data points). In some of the calculations reported below, we allow K00137.03 to interact dynamically, but we include only its first and last observed transits, at $t=2454955.99237 \pm 0.00823$ and $2455530.77178 \pm 0.00174$ (two data points), as a way of keeping its period and phase fixed at observed values. We have measured all of its transit times, but we do not report or analyze them here: their individual signal to noise is low, they are not apparently constant, and we have not found a consistent dynamical solution 
Table 5

Transit Times for Kepler-18c = K00137.01

\begin{tabular}{|c|c|c|c|}
\hline Cycle $^{\mathrm{a}}$ & BJD-2455000.0 & $O-C$ & $\sigma$ \\
\hline-27.0 & -39.23132 & +0.00343 & 0.00141 \\
\hline-26.0 & -31.59238 & +0.00077 & 0.00128 \\
\hline-25.0 & -23.94944 & +0.00212 & 0.00134 \\
\hline-24.0 & -16.30829 & +0.00168 & 0.00125 \\
\hline-23.0 & -8.66703 & +0.00134 & 0.00202 \\
\hline-21.0 & 6.61139 & -0.00342 & 0.00161 \\
\hline-20.0 & 14.25400 & -0.00241 & 0.00155 \\
\hline-19.0 & 21.89543 & -0.00257 & 0.00149 \\
\hline-18.0 & 29.53633 & -0.00327 & 0.00135 \\
\hline-17.0 & 37.17764 & -0.00355 & 0.00131 \\
\hline-16.0 & 44.82280 & +0.00002 & 0.00146 \\
\hline-15.0 & 52.46001 & -0.00436 & 0.00107 \\
\hline-14.0 & 60.10428 & -0.00169 & 0.00115 \\
\hline-13.0 & 67.74533 & -0.00223 & 0.00108 \\
\hline-12.0 & 75.39019 & +0.00103 & 0.00145 \\
\hline-11.0 & 83.02837 & -0.00238 & 0.00144 \\
\hline-10.0 & 90.67043 & -0.00191 & 0.00140 \\
\hline-9.0 & 98.31334 & -0.00059 & 0.00102 \\
\hline-8.0 & 105.95509 & -0.00044 & 0.00105 \\
\hline-7.0 & 113.59742 & +0.00030 & 0.00148 \\
\hline-6.0 & 121.23989 & +0.00117 & 0.00098 \\
\hline-5.0 & 128.88188 & +0.00157 & 0.00108 \\
\hline-4.0 & 136.52252 & +0.00062 & 0.00102 \\
\hline-3.0 & 144.16670 & +0.00320 & 0.00088 \\
\hline-2.0 & 151.80782 & +0.00273 & 0.00137 \\
\hline-1.0 & 159.44986 & +0.00318 & 0.00113 \\
\hline 0.0 & 167.09286 & +0.00459 & 0.00096 \\
\hline 1.0 & 174.73457 & +0.00470 & 0.00097 \\
\hline 2.0 & 182.37715 & +0.00568 & 0.00131 \\
\hline 3.0 & 190.01671 & +0.00365 & 0.00106 \\
\hline 4.0 & 197.65742 & +0.00277 & 0.00109 \\
\hline 5.0 & 205.30025 & +0.00400 & 0.00117 \\
\hline 6.0 & 212.94064 & +0.00281 & 0.00101 \\
\hline 7.0 & 220.57949 & +0.00006 & 0.00111 \\
\hline 8.0 & 228.22281 & +0.00178 & 0.00101 \\
\hline 9.0 & 235.86365 & +0.00103 & 0.00106 \\
\hline 10.0 & 243.50537 & +0.00116 & 0.00107 \\
\hline 11.0 & 251.14597 & +0.00017 & 0.00097 \\
\hline 12.0 & 258.78515 & -0.00224 & 0.00092 \\
\hline 13.0 & 266.42783 & -0.00116 & 0.00115 \\
\hline 14.0 & 274.06799 & -0.00259 & 0.00118 \\
\hline 15.0 & 281.70882 & -0.00335 & 0.00110 \\
\hline 16.0 & 289.35100 & -0.00277 & 0.00079 \\
\hline 17.0 & 296.99249 & -0.00288 & 0.00088 \\
\hline 18.0 & 304.63304 & -0.00392 & 0.00083 \\
\hline 19.0 & 312.27505 & -0.00350 & 0.00093 \\
\hline 20.0 & 319.91502 & -0.00512 & 0.00090 \\
\hline 21.0 & 327.56008 & -0.00165 & 0.00089 \\
\hline 22.0 & 335.19905 & -0.00427 & 0.00093 \\
\hline 23.0 & 342.84138 & -0.00355 & 0.00084 \\
\hline 24.0 & 350.48436 & -0.00215 & 0.00103 \\
\hline 25.0 & 358.12585 & -0.00226 & 0.00097 \\
\hline 26.0 & 365.76604 & -0.00367 & 0.00086 \\
\hline 27.0 & 373.41078 & -0.00052 & 0.00095 \\
\hline 28.0 & 381.05287 & -0.00001 & 0.00101 \\
\hline 29.0 & 388.69546 & +0.00098 & 0.00089 \\
\hline 30.0 & 396.33836 & +0.00228 & 0.00090 \\
\hline 31.0 & 403.97897 & +0.00130 & 0.00096 \\
\hline 32.0 & 411.62239 & +0.00313 & 0.00104 \\
\hline 33.0 & 419.26374 & +0.00288 & 0.00090 \\
\hline 34.0 & 426.90502 & +0.00256 & 0.00100 \\
\hline 35.0 & 434.54873 & +0.00468 & 0.00112 \\
\hline 36.0 & 442.18944 & +0.00381 & 0.00091 \\
\hline 37.0 & 449.82907 & +0.00184 & 0.00106 \\
\hline 38.0 & 457.46935 & +0.00052 & 0.00097 \\
\hline
\end{tabular}

Table 5

(Continued)

\begin{tabular}{cccc}
\hline \hline Cycle $^{\mathrm{a}}$ & BJD-2455000.0 & $O-C$ & $\sigma$ \\
\hline 39.0 & 465.11288 & +0.00246 & 0.00092 \\
40.0 & 472.75469 & +0.00268 & 0.00103 \\
41.0 & 480.39522 & +0.00161 & 0.00117 \\
42.0 & 488.03539 & +0.00020 & 0.00101 \\
43.0 & 495.67681 & +0.00002 & 0.00100 \\
44.0 & 503.31876 & +0.00037 & 0.00092 \\
45.0 & 510.95872 & -0.00125 & 0.00091 \\
46.0 & 518.60144 & -0.00014 & 0.00088 \\
47.0 & 526.24067 & -0.00249 & 0.00114 \\
48.0 & 533.88235 & -0.00241 & 0.00112 \\
\hline
\end{tabular}

Note. ${ }^{a} P=7.64159$ days, $T_{0}=2455167.08828$.

Table 6

Transit Times for Kepler-18d = K00137.02

\begin{tabular}{|c|c|c|c|}
\hline Cycle $^{\mathrm{a}}$ & BJD-2455000.0 & $O-C$ & $\sigma$ \\
\hline-14.0 & -38.84989 & -0.00318 & 0.00101 \\
\hline-13.0 & -23.98927 & -0.00144 & 0.00090 \\
\hline-12.0 & -9.12888 & +0.00008 & 0.00081 \\
\hline-11.0 & 5.73040 & +0.00048 & 0.00086 \\
\hline-10.0 & 20.59242 & +0.00362 & 0.00115 \\
\hline-9.0 & 35.44894 & +0.00126 & 0.00074 \\
\hline-8.0 & 50.30774 & +0.00119 & 0.00091 \\
\hline-7.0 & 65.16644 & +0.00101 & 0.00078 \\
\hline-6.0 & 80.02557 & +0.00126 & 0.00121 \\
\hline-5.0 & 94.88445 & +0.00127 & 0.00068 \\
\hline-4.0 & 109.74201 & -0.00005 & 0.00065 \\
\hline-3.0 & 124.59986 & -0.00107 & 0.00080 \\
\hline-2.0 & 139.45743 & -0.00238 & 0.00065 \\
\hline-1.0 & 154.31562 & -0.00307 & 0.00067 \\
\hline 0.0 & 169.17495 & -0.00261 & 0.00070 \\
\hline 2.0 & 198.89361 & -0.00170 & 0.00081 \\
\hline 3.0 & 213.75079 & -0.00341 & 0.00085 \\
\hline 4.0 & 228.61267 & -0.00040 & 0.00079 \\
\hline 5.0 & 243.47039 & -0.00155 & 0.00081 \\
\hline 6.0 & 258.33093 & +0.00011 & 0.00068 \\
\hline 7.0 & 273.19074 & +0.00104 & 0.00071 \\
\hline 8.0 & 288.05139 & +0.00282 & 0.00069 \\
\hline 9.0 & 302.91049 & +0.00304 & 0.00061 \\
\hline 10.0 & 317.77010 & +0.00377 & 0.00065 \\
\hline 11.0 & 332.62850 & +0.00330 & 0.00061 \\
\hline 12.0 & 347.48637 & +0.00229 & 0.00069 \\
\hline 13.0 & 362.34423 & +0.00127 & 0.00072 \\
\hline 14.0 & 377.20286 & +0.00103 & 0.00070 \\
\hline 15.0 & 392.06096 & +0.00025 & 0.00063 \\
\hline 16.0 & 406.91891 & -0.00068 & 0.00061 \\
\hline 17.0 & 421.77755 & -0.00091 & 0.00061 \\
\hline 18.0 & 436.63489 & -0.00245 & 0.00063 \\
\hline 19.0 & 451.49447 & -0.00175 & 0.00067 \\
\hline 20.0 & 466.35351 & -0.00158 & 0.00063 \\
\hline 21.0 & 481.21293 & -0.00104 & 0.00066 \\
\hline 22.0 & 496.07232 & -0.00052 & 0.00067 \\
\hline 23.0 & 510.93180 & +0.00008 & 0.00061 \\
\hline
\end{tabular}

Note. ${ }^{\mathrm{a}} P=14.85888$ days, $T_{0}=2455169.17756$.

to date. Perhaps future work will show a fourth (non-transiting) planet is required to fit the transit times of Kepler-18b. In the meantime we proceed with fitting the three transiting planets with a focus on the transit timing constraints for Kepler-18c and Kepler-18d.

By fitting only the transit times, allowing $P$ and $T_{0}$ for each of the three planets to vary (six free parameters), and setting 

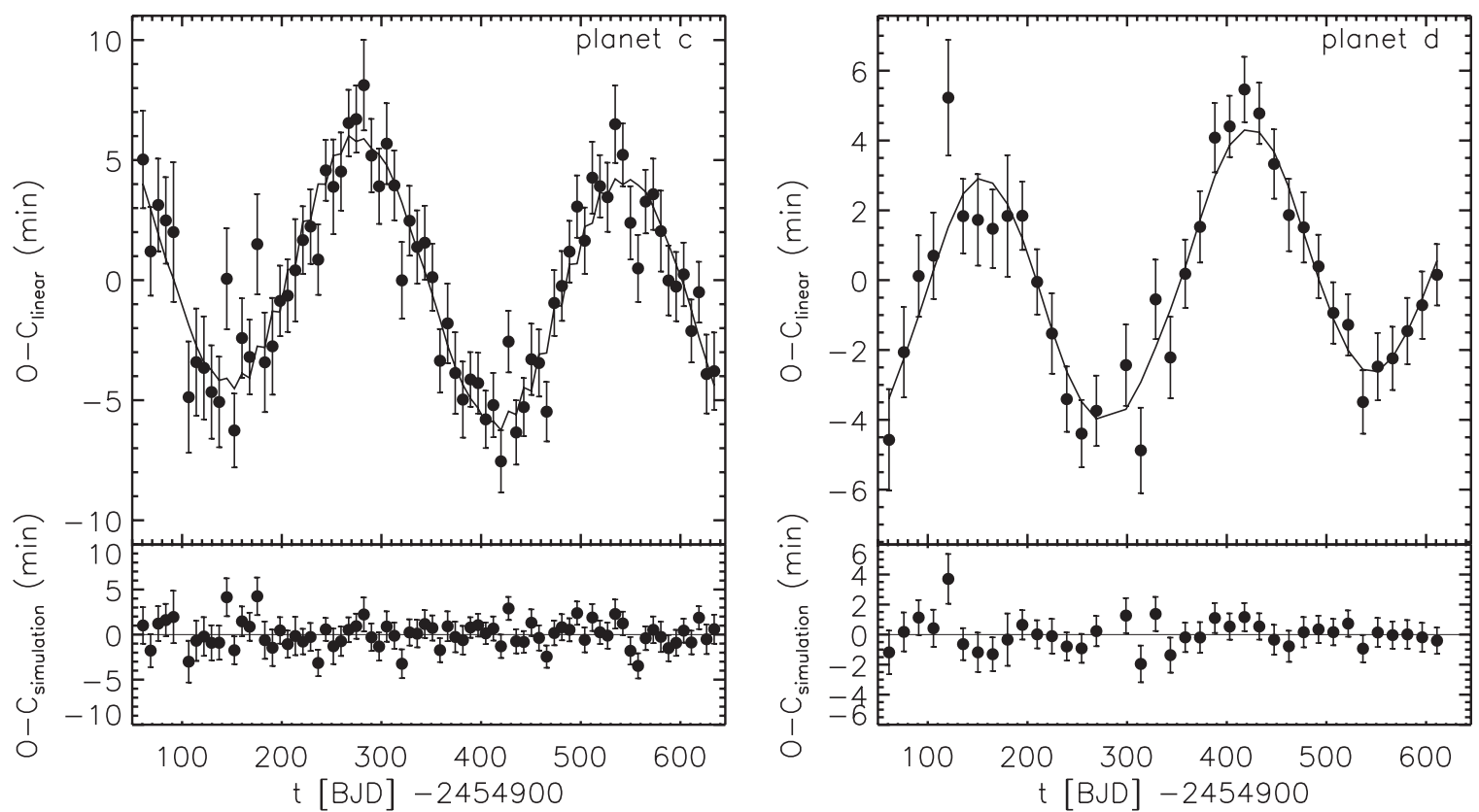

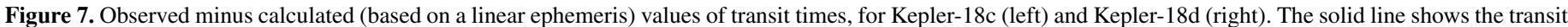
times calculated using a dynamical model. The lower panels show the residuals of the measurements from the model.

dynamical interactions to zero, we find $\chi^{2}=750.6$ for 114 transit time measurements. This is clearly an unacceptable fit, and the obvious timing patterns call for a dynamical model. The observed (" $O$ ") residuals to this calculated (" $C$ ") ephemeris are called the $O-C$ values and are plotted in Figure 7 along with the preferred dynamical model described below. As in the case of Kepler-11b/c, we clearly see that the source of the variations of transit times for Kepler-18c and Kepler-18d is their nearly 2:1 resonance. The expected variation occurs on a timescale:

$$
P_{\mathrm{TTV}}=1 /\left(2 / P_{d}-1 / P_{c}\right)=268 \text { days, }
$$

the time it takes the line of conjunctions to sweep around inertial space, through both the line of sight and the apsidal lines of these planets (on the approximation that precession can be ignored on this timescale); see Agol et al. (2005). In Figure 8, we plot the periodogram of the $O-C$ values for Kepler-18c and Kepler$18 \mathrm{~d}$. The peaks occur at $1 /(260.4 \pm 3.3$ days $)$ and $1 /(265.1 \pm$ 4.7 days) for Kepler-18c and Kepler-18d, respectively, very close to the simple expectation given above, which uniquely identifies the dynamical mechanism for TTVs.

Before moving on to a full solution, we tried fitting the radial velocities (14 data points, Table 3 ) with the above-determined periods and phases, with circular orbits, allowing only the planetary masses to vary. The solution, listed in the second row of Table 8, was $\left[m_{b}, m_{c}, m_{d}\right]=[12 \pm 5,15 \pm 5,28 \pm 7] M_{\oplus}$; the $\chi^{2}=9.7$ for 10 degrees of freedom (14 RV data points, minus $3 \mathrm{~K}$-amplitudes, minus a constant $\mathrm{RV}$ offset). These masses are well within the $1 \sigma$ error bars of the MCMC solution to the light curve and RVs presented in Section 4. Therefore, this model is sufficient to explain the radial velocities, and each planet is detected, but only marginally so for planet $b$. In particular, if we hold the mass of $\mathrm{b}$ at zero, the masses of the others become $\left[m_{c}, m_{d}\right]=[18 \pm 5,24 \pm 7] M_{\oplus}$ and the $\chi^{2}=15.6$ for 10 degrees of freedom. These values are within about $0.5 \sigma$ of the masses determined from the joint MCMC and RV solution presented earlier in Section 4.

We may also attempt to constrain the masses and orbital elements using only the transit times. Naturally, this requires

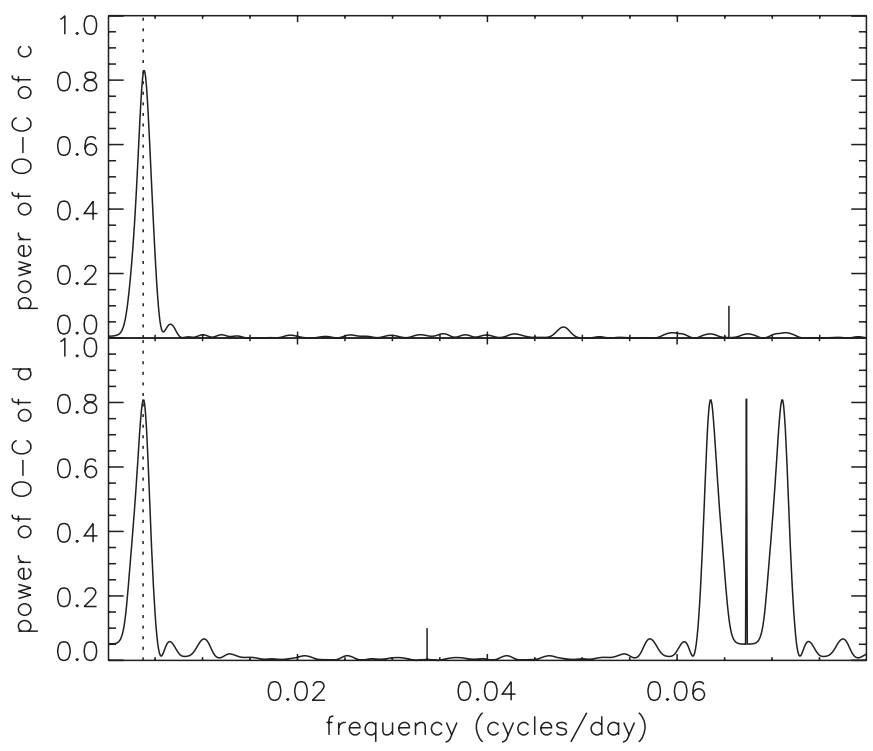

Figure 8. Periodograms (fractional $\chi^{2}$ reduction as a function of frequency; Zechmeister \& Kürster 2009) of the $O-C$ values shown in Figure 7. The dashed line shows the expected timescale from Equation (1), showing excellent agreement. The large tick marks show the Nyquist frequency $1 /\left(2 P_{\text {orbital }}\right)$ beyond which the periodogram holds no additional information.

full dynamical integrations, in which the planets cannot remain on circular orbits. However, for planet $\mathrm{b}$ we assumed a circular orbit at the dynamical epoch, since we are not attempting to fit its transit time variations. All of the other orbital parameters and masses were free to vary. The resulting $\chi^{2}$ is 88.5 for 101 degrees of freedom (114 transit times, minus 5 parameters for planets c and d, minus 3 for K00137.03), which is quite acceptable. To be compared with the RV solution, the solved-for masses were $\left[m_{b}, m_{c}, m_{d}\right]=[18 \pm 9,17.3 \pm 1.7,15.8 \pm 1.3] M_{\oplus}$, shown in the third row of Table 8. These solutions had extremely low eccentricities $(e<0.003)$ for Kepler-18c and Kepler-18d. As above, the innermost planet is only very marginally detected. In contrast to the RV solution, however, the masses of the 
Table 7

Osculating Jacobian Elements, at Epoch 2455168.0 [BJD], for the Three-planet TTV Dynamical Solution

\begin{tabular}{lcccc}
\hline \hline Planet & $\begin{array}{c}\text { Period } \\
\text { (days) }\end{array}$ & $\begin{array}{c}T_{0} \\
\text { (days) }\end{array}$ & $e \cos \omega$ & $e \sin \omega$ \\
\hline $\mathrm{b}$ & $3.504674 \pm 0.000054$ & $266.276996 \pm 0.005453$ & 0 & 0 \\
$\mathrm{c}$ & $7.641039 \pm 0.000087$ & $267.092502 \pm 0.000262$ & $0.000291 \pm 0.000079$ & $0.000173 \pm 0.000233$ \\
$\mathrm{~d}$ & $14.860509 \pm 0.000148$ & $269.174850 \pm 0.000253$ & $-0.000076 \pm 0.000019$ & $0.000516 \pm 0.000450$ \\
\hline
\end{tabular}

Table 8

Masses and Densities of the Planets in the Kepler-18 System

\begin{tabular}{lcccc}
\hline \hline Method & & $\begin{array}{c}\text { Kepler-18b } \\
\text { K00137.03) }\end{array}$ & $\begin{array}{c}\text { Kepler-18c } \\
(\text { K00137.01) }\end{array}$ & $\begin{array}{c}\text { Kepler-18d } \\
(\text { K00137.02) }\end{array}$ \\
\hline MCMC (light curve + RV) solution & $\left(M_{\oplus}\right)$ & $13.4 \pm 5.8$ & $16.9 \pm 6.1$ & $29.9 \pm 8.8$ \\
RV + transit time $P$ and $T_{0}$ & $\left(M_{\oplus}\right)$ & $12 \pm 5$ & $15 \pm 5$ & $28 \pm 7$ \\
TTV dynamical model & $\left(M_{\oplus}\right)$ & $18 \pm 9$ & $17.3 \pm 1.7$ & $15.8 \pm 1.3$ \\
TTV + RV dynamical model (adopted values) & $\left(M_{\oplus}\right)$ & $6.9 \pm 3.4$ & $17.3 \pm 1.9$ & $16.4 \pm 1.4$ \\
\hline Density from adopted mass & $\left(\mathrm{g} \mathrm{cm}^{-3}\right)$ & $4.9 \pm 2.4$ & $0.59 \pm 0.07$ & $0.27 \pm 0.03$ \\
\hline
\end{tabular}

interacting planets are very precisely pinned down by the large variations seen in Figure 7.

Finally, we generated a joint solution to the transit times and radial velocities. Graphically, this solution is given by Figure 7 . A $\chi^{2}=103.4$ for 114 degrees of freedom is achieved, an excellent fit to the data. The orbital parameters and their formal errors (the output of the Levenberg-Marquardt algorithm) are given in Table 7, and the planetary masses are given in the fourth row of Table 8 .

In the previous subsection we found no drift in the inclinations of the three planets. We can use the $3 \sigma$ upper limits on $|\Delta i / \Delta E|$ of $[37,70,33] \times 10^{-4}$ degrees per epoch for Kepler-18b, c, and $\mathrm{d}$ to place limits on their mutual inclination (Miralda-Escudé 2002). To do this, we measure the value of $|\Delta i / \Delta E|$ seen in numerical simulation, which depends nearly linearly on the difference in nodal angle on the sky of two planets (Ballard et al. 2010). Taking the masses and orbits from the best-fit TTV/RV solution above, we simulated Kepler-18c and d with $1^{\circ}$ (and $\left.10^{\circ}\right)$ of mutual inclination, we find $|\Delta i / \Delta E|_{c}=1.8 \times 10^{-4} \mathrm{de}-$ gree per epoch $\left(16 \times 10^{-4}\right.$ degree per epoch $)$ and $|\Delta i / \Delta E|_{d}=$ $2.9 \times 10^{-4}$ degree per epoch $\left(26 \times 10^{-4}\right.$ degree per epoch $)$. The interaction between Kepler-18b and $\mathrm{c}$ is also potentially observable; simulating their orbits with $1^{\circ}\left(10^{\circ}\right)$ of mutual inclination, we find $|\Delta i / \Delta E|_{b}=1.3(11) \times 10^{-4}$ degree per epoch and $|\Delta i / \Delta E|_{c}=0.82(7.4) \times 10^{-4}$ degree per epoch. The interaction between Kepler-18b and d is considerably weaker, with a drift of $\sim 2 \times 10^{-4}$ degree per epoch even for $10^{\circ}$ mutual inclination; therefore we ignore it when setting mutual inclination limits. To find the $3 \sigma$ upper limit to the nodal difference of $\mathrm{c}$ and $\mathrm{d}$, we compare the $10^{\circ}$ calculated value of $|\Delta i / \Delta E|_{d}$ to its observational $3 \sigma$ upper limit, so we find $\left|\Omega_{c}-\Omega_{d}\right|<13^{\circ}$. The inclination difference in the complementary direction is only $i_{\mathrm{d}}-i_{\mathrm{c}}=0.40 \pm 0.24$, so the limit on the true mutual inclination is $i_{\mathrm{cd}}<13^{\circ}$. The nodal difference of $\mathrm{b}$ and $\mathrm{c}$ is more poorly constrained, as the calculated value of $|\Delta i / \Delta E|$ for both planets in the $10^{\circ}$ mutual inclination simulation is small compared to its observational $3 \sigma$ upper limit. Thus, a moderate $\left(\sim 20^{\circ}\right)$ mutual inclination is permissible, which would be large enough to affect the timing fits. In that case, the model should self-consistently fit the radial velocities, transit times, and transit durations. However, we defer dynamical interpretation of the mutual inclination of the inner planet to the others until more data are gathered, which should tighten the limit.

\section{BLENDER ANALYSIS OF K00137.03}

The lack of a clear dynamical confirmation of the nature of K00137.03 requires us to examine the wide variety of astrophysical false positives (blends) that might mimic the photometric transit, and to assess their a priori likelihood compared to that of a true planet. For this we apply the BLENDER technique described by Torres et al. $(2004,2011)$ with further developments as reported by Fressin et al. (2011). BLENDER uses the detailed shape of the transit light curve to weed out scenarios that lead to the wrong shape for a transit. The kinds of false positives we are concerned with here include background or foreground eclipsing binaries blended with the target, as well as physically associated eclipsing binaries, which generally cannot be resolved in high angular resolution imaging. In each case the pair of eclipsing objects can also be a star transited by a larger planet. Briefly, BLENDER simulates a very large number of light curves resulting from these blend scenarios with a range of stellar (or planetary) parameters, and compares them to the Kepler photometry in a $\chi^{2}$ sense. Blends providing poor fits are considered to be ruled out, enabling us to place constraints on the kinds of objects composing the eclipsing pair that yield viable blends, including their sizes or masses, as well as other properties of the blend such as the overall brightness and color, and even the eccentricities $(e)$ of the orbits. We refer the reader to the above references for details. Following the nomenclature in those sources, the objects in the eclipsing pair are designated as the "secondary" and "tertiary," and the target itself is the "primary." Stellar properties are drawn from model isochrones.

Simulations with BLENDER indicate that background eclipsing binaries with two stellar components can only produce viable false positives if they are restricted to a narrow range of masses for the secondaries (approximately $0.8 \leqslant M_{2} / M_{\odot} \leqslant 1.3$ ), as well as a limited interval in brightness ( $K p$ magnitude) relative to the target $(4 \leqslant \Delta K p \leqslant 7$ ). This is illustrated in Figure 9, in which we show the $\chi^{2}$ landscape from BLENDER for all blend fits of this kind. Regions outside the $3 \sigma$ contour correspond to scenarios with transit shapes that do not provide acceptable fits to the Kepler photometry, i.e., fits that are much worse than a true planet fit. These configurations are therefore excluded.

For blends involving a background/foreground star transited by a larger planet, there is in principle a wide range of allowed masses (spectral types) for the secondary stars, as shown in 


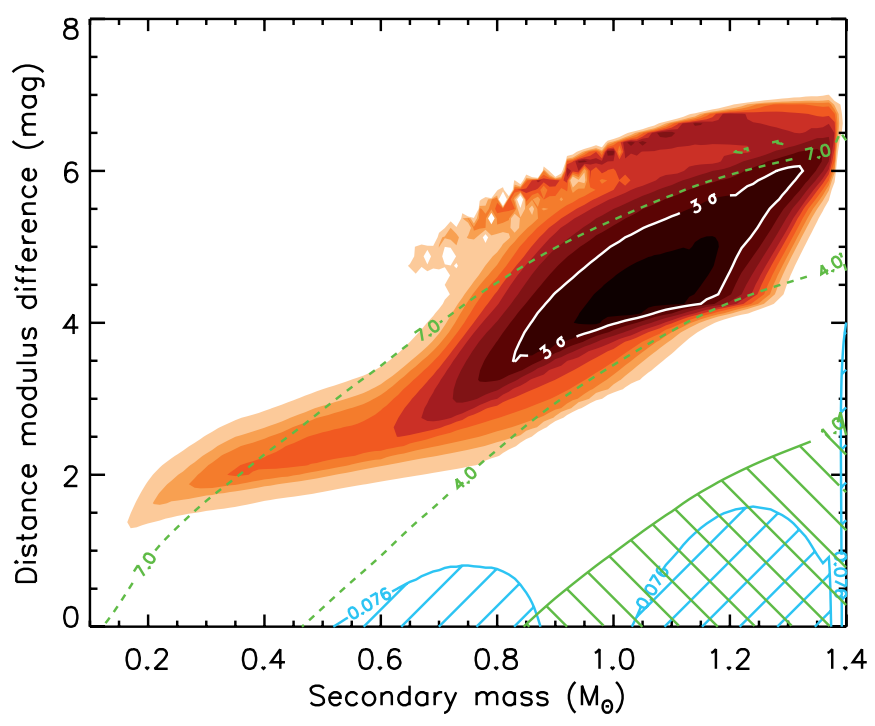

Figure 9. Map of the Kepler-18b (K00137.03) $\chi^{2}$ surface (goodness of fit) for blends involving background eclipsing binaries with stellar tertiaries and arbitrary orbital eccentricities. The vertical axis represents the linear separation between the background binary and the primary, cast for convenience in terms of the difference in the distance modulus. Only blends within the solid white contour match the Kepler light curve within acceptable limits $(3 \sigma$, where $\sigma$ is the significance level of the $\chi^{2}$ difference compared to a transiting planet model; see Fressin et al. 2011). Other concentric colored areas represent increasingly worse fits $(4 \sigma, 5 \sigma$, etc.), and correspond to blends we consider to be ruled out. Dashed green lines are labeled with the magnitude difference $\Delta K p$ between the blended binary and the primary, and encompass the brightness range allowed by BLENDER ( $4 \leqslant \Delta K p \leqslant 7)$. Blends with eclipsing binaries bright enough to be detected spectroscopically ( $\Delta K p \leqslant 1 \mathrm{mag}$ ) are indicated with the hatched region below the solid green line, but are already ruled out by BLENDER. Similarly with the blue hatched areas that mark blends that are either too red or too blue compared to the measured color (see the text and Figure 10). When further constraining these blends to have realistic eccentricities ( $e \leqslant 0.1$; see the text), we find that all of them are excluded by BLENDER.

(A color version of this figure is available in the online journal.)

Figure 10. However, other constraints available for Kepler-18 strongly limit the number of these false positives. In particular, by comparing the predicted $r-K_{s}$ color of each blend against the measured color of the star from the KIC $\left(r-K_{s}=\right.$ $1.723 \pm 0.031$; Brown et al. 2011), we find that many of the smaller-mass secondaries are ruled out because the blends would be much too red compared to the known color index of Kepler-18 (by more than $3 \sigma$ ). Others are excluded because the secondary star would be very bright (within $1 \mathrm{mag}$ of the target, or in some cases even brighter than the target), and would have been noticed spectroscopically if unresolved in our AO or speckle imaging. This removes many but not all blends of this kind.

For eclipsing binaries that are physically associated with the target (in a hierarchical triple star configuration), we find that the blend light curves invariably have the wrong shape to mimic a true transiting planet signal, for any combination of stellar parameters for the secondary and tertiary. Either the depth, duration, or steepness of the ingress/egress phases of the transits provide a poor match to the Kepler photometry. These scenarios are therefore all excluded. On the other hand, if we allow the tertiaries to be planets, then we do find a variety of secondary masses that can produce viable blends when transited by a planet of the appropriate size. The $\chi^{2}$ map for this general case appears in Figure 11, and shows the range of radii permitted for the tertiaries, as well as the interval of secondary masses that yield suitable blends.

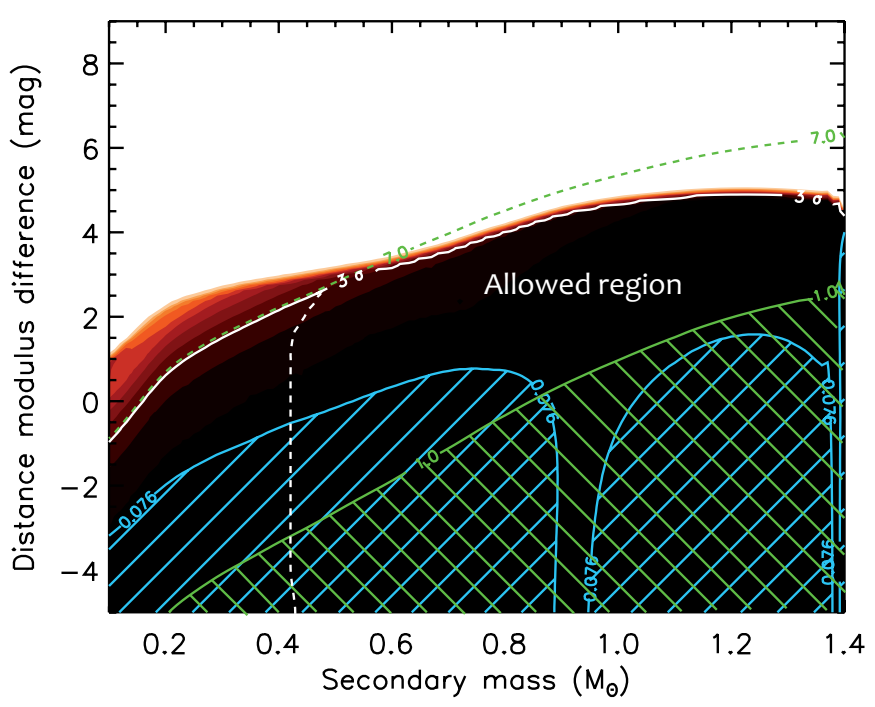

Figure 10. Similar to Figure 9 (and with the same color scheme) for Kepler-18b (K00137.03) blends involving background or foreground stars transited by a larger planet. The blue hatched regions correspond to blends that are too red (left) or too blue (right) compared to the measured $r-K_{s}$ color of Kepler-18, and are thus ruled out. When blends are restricted to realistic orbital eccentricities $(e \leqslant 0.3)$, many of the later-type secondaries are excluded (dashed $3 \sigma$ contour) The combination of the brightness (green hatched area) and color constraints leaves only a reduced area of parameter space ("allowed region") where blends are a suitable alternative to a transiting planet model. All of these scenarios have $\Delta K p<7.0$ (dashed green line)

(A color version of this figure is available in the online journal.)

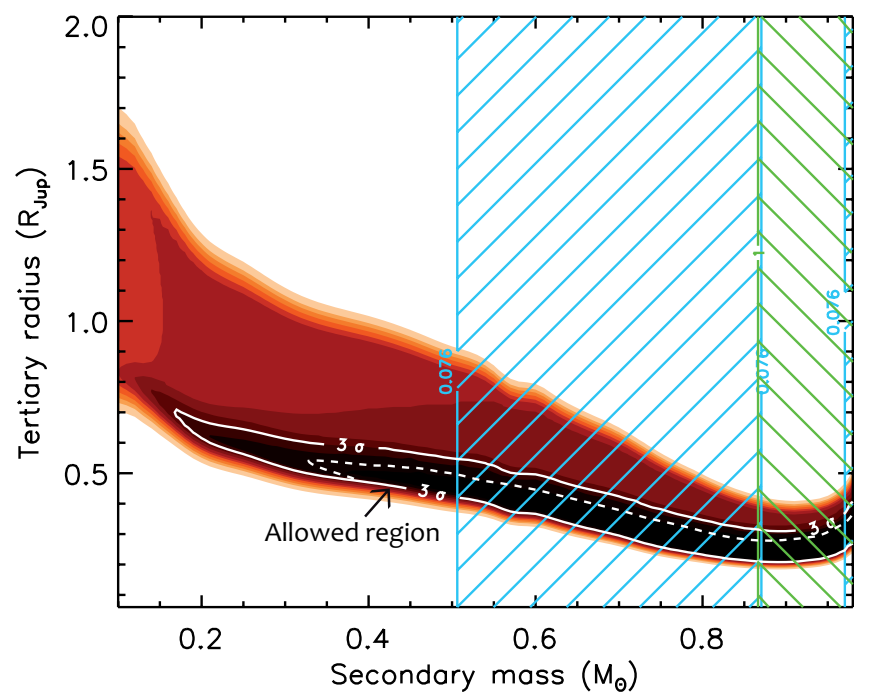

Figure 11. Similar to Figure 9 for Kepler-18b (K00137.03) for the case of hierarchical triple systems in which the secondary is transited by a planet. After taking into account the constraints on the $r-K_{s}$ color and brightness (blue and green hatched regions, respectively), only secondary stars with $M_{2} \leqslant 0.5 M_{\odot}$ lead to blend light curves that match the observations. Further restriction to realistic orbital eccentricities ( $e \leqslant 0.3$; see the text) leads to a slightly smaller $3 \sigma$ contour (dashed).

(A color version of this figure is available in the online journal.)

The duration of a transit is set by the length of the chord traversed by the tertiary and the tangential velocity of the tertiary during the event. The chord length, in turn, depends on the size of the secondary and the impact parameter. Therefore, the measured duration of a transit provides a strong constraint on the allowed sizes for the secondary stars (or equivalently, their masses or spectral types). In the above BLENDER simulations, we have placed no restriction on the orbital eccentricities of the star+star or star+planet pairs that can be blended with 
Table 9

Blend Frequency Estimate for K00137.03

\begin{tabular}{|c|c|c|c|c|c|c|}
\hline \multirow[b]{2}{*}{$\begin{array}{l}\text { Kp Range } \\
(\mathrm{mag}) \\
(1)\end{array}$} & \multirow[b]{2}{*}{$\begin{array}{l}\Delta K p \\
(\mathrm{mag}) \\
(2)\end{array}$} & \multicolumn{5}{|c|}{ Blends Involving Planetary Tertiaries } \\
\hline & & $\begin{array}{c}\text { Stellar } \\
\text { Mass Range } \\
\left(M_{\odot}\right) \\
(3)\end{array}$ & $\begin{array}{l}\text { Stellar Density } \\
\left.\text { (per } \mathrm{deg}^{2}\right) \\
(4)\end{array}$ & $\begin{array}{l}\rho_{\max } \\
(") \\
(5)\end{array}$ & $\begin{array}{c}\text { Stars } \\
\left(\times 10^{-6}\right) \\
(6)\end{array}$ & $\begin{array}{c}\text { Transiting Planets } \\
0.32-1.96 R_{\text {Jup }}, f_{\text {planet }}=0.24 \% \\
\left(\times 10^{-6}\right) \\
(7)\end{array}$ \\
\hline $13.5-14.0$ & 0.5 & $\ldots$ & $\ldots$ & $\ldots$ & $\ldots$ & $\ldots$ \\
\hline $14.0-14.5$ & 1.0 & $\ldots$ & $\ldots$ & $\ldots$ & $\ldots$ & $\ldots$ \\
\hline $14.5-15.0$ & 1.5 & $0.87-1.40$ & 862 & 0.08 & 1.34 & 0.003 \\
\hline $15.0-15.5$ & 2.0 & $0.82-1.40$ & 1377 & 0.11 & 4.04 & 0.010 \\
\hline $15.5-16.0$ & 2.5 & $0.78-1.40$ & 2094 & 0.13 & 8.58 & 0.021 \\
\hline $16.0-16.5$ & 3.0 & $0.72-1.40$ & 3053 & 0.16 & 18.9 & 0.045 \\
\hline $16.5-17.0$ & 3.5 & $0.43-1.40$ & 4341 & 0.20 & 42.1 & 0.101 \\
\hline $17.0-17.5$ & 4.0 & $0.43-1.40$ & 5873 & 0.24 & 82.0 & 0.197 \\
\hline $17.5-18.0$ & 4.5 & $0.43-1.32$ & 7599 & 0.32 & 189 & 0.454 \\
\hline $18.0-18.5$ & 5.0 & $0.43-1.25$ & 9399 & 0.40 & 365 & 0.876 \\
\hline $18.5-19.0$ & 5.5 & $0.43-1.09$ & 10819 & 0.56 & 822 & 1.973 \\
\hline $19.0-19.5$ & 6.0 & $0.43-1.01$ & 11988 & 0.64 & 1190 & 2.856 \\
\hline $19.5-20.0$ & 6.5 & $0.43-0.92$ & 12585 & 0.80 & 1952 & 4.685 \\
\hline $20.0-20.5$ & 7.0 & $0.43-0.61$ & 3373 & 0.88 & 633 & 1.519 \\
\hline $20.5-21.0$ & 7.5 & $\ldots$ & $\ldots$ & $\ldots$ & $\ldots$ & $\ldots$ \\
\hline $21.0-21.5$ & 8.0 & $\ldots$ & $\ldots$ & $\ldots$ & $\ldots$ & $\ldots$ \\
\hline \multicolumn{2}{|c|}{ Totals } & & & & 5308 & 12.7 \\
\hline \multicolumn{7}{|c|}{ Blend frequency from hierarchical triples (see the text) $=4.4 \times 10^{-6}$} \\
\hline \multicolumn{7}{|c|}{ Total frequency $(\mathrm{BF})=(12.7+4.4) \times 10^{-6}=17.1 \times 10^{-6}$} \\
\hline
\end{tabular}

Note. Magnitude bins with no entries correspond to brightness ranges in which BLENDER excludes all blends.

the target. When the orbits are permitted to be eccentric, the tangential velocity of the tertiary during transit can be significantly different from the circular case, and this allows a much larger range of secondary sizes than would otherwise be possible. In particular, chance alignments with later-type stars transited by a planet near apoastron become viable as blends, and represent a good fraction of the false positives shown in Figures 10 and 11. Some blends involving larger secondaries transited at periastron can also provide acceptable fits.

We note, however, that the period of K00137.03 is relatively short (3.5 days), and very large eccentricities, such as some of our simulated blends have, are unlikely as they would be expected to be damped by tidal forces (see Mazeh 2008). Indeed, among binaries with main-sequence primary stars that are similar to Kepler-18 (solar-type or later), all systems under 3.5 days have essentially circular orbits (see, e.g., Halbwachs et al. 2003; Raghavan et al. 2010). We may take $e=0.1$ as a conservative upper limit. Similarly, among the known transiting planets with periods of 3.5 days or shorter and host stars of any spectral type, none are found to have eccentricities as large as $e=0.3$. When constraining the false positives for K00137.03 to be within these eccentricity limits, we find that all background eclipsing binaries (stellar tertiaries) are easily excluded as they all require fairly eccentric orbits in order to match the observed duration. Additionally, the numbers of blends involving star+planet pairs in the foreground/background or in hierarchical triple systems are considerably reduced when restricting the eccentricities, although many remain that we cannot rule out. In the following we assess their frequency, and compare it with the expected frequency of transiting planets.

\subsection{Validating K00137.03}

The a priori frequency of stars in the background or foreground of the target that are orbited by a transiting planet and are capable of mimicking the K00137.03 signal may be estimated from the number density of stars in the vicinity of Kepler-18, the area around the target in which such stars would go undetected in our high-resolution imaging, and the frequency of transiting planets with the appropriate characteristics. To obtain the number density (stars per square degree) we make use of the Galactic structure models of Robin et al. (2003), and we perform this calculation in half-magnitude bins, as shown in Table 9. For each bin we further restrict the star counts using the constraints on the mass of the secondaries supplied by BLENDER (see Figure 10), and the eccentricity limit for transiting planets discussed above $(e \leqslant 0.3)$. These mass ranges are listed in Column 3 , and the resulting densities appear in Column 4. Bins with no entries correspond to brightness ranges excluded by BLENDER. The maximum angular separation $\left(\rho_{\max }\right)$ at which stars of each brightness would escape detection in our $\mathrm{AO} /$ speckle imaging is shown in Column 5 (see Table 1 and Section 3.2). The result for the number of stars in each magnitude bin is given in Column 6 , in units of $10^{-6}$.

To estimate the frequency of transiting planets that might be expected to orbit these stars (and lead to a false positive), we rely on the results from Borucki et al. (2011b), who reported a total of 1235 planet candidates among the 156,453 Kepler targets observed during the first four months of the mission. These signals have not yet been confirmed to be caused by planets, and therefore remain candidates until they can be thoroughly followed up. However, the rate of false positives in this sample is expected to be quite small $(\sim 10 \%$ or less; see Morton \& Johnson 2011), so our results will not be significantly affected by the assumption that all of the candidates are planets. We further assume that the census of Borucki et al. (2011b) is largely complete. After accounting for the additional BLENDER constraint on the range of planet sizes for blends of this kind (tertiaries of $0.32-1.96 R_{\mathrm{Jup}}$ ), we find that the transiting planet 
frequency is $f_{\text {planet }}=374 / 156,453=0.0024$. Multiplying this frequency by the star counts in Column 6 of Table 9, we arrive at the blend frequencies listed in Column 7 , which are added up in the "Totals" line of the table $\left(12.7 \times 10^{-6}\right)$.

Next we address the frequency of hierarchical triples, that is, physically associated companions to the target that are orbited by a larger transiting planet able to mimic the signal. The rate of occurrence of this kind of false positive may be estimated by considering the overall frequency of binary stars (34\% according to Raghavan et al. 2010) along with constraints on the mass range of such companions and how often they would be orbited by a transiting planet of the right size $\left(0.43-0.53 R_{\text {Jup }}\right.$; see Figure 11). The mass constraints include not only those coming directly from BLENDER, but also take into consideration the color and brightness limits mentioned earlier. We performed this calculation in a Monte Carlo fashion, drawing the secondary stars from the mass ratio distribution reported by Raghavan et al. (2010), and the transiting planet eccentricities from the actual distribution of known transiting planets (http://exoplanet.eu/), with repetition. Planet frequencies in the appropriate radius range were taken as before from Borucki et al. (2011b). The result is a frequency of hierarchical triples of $4.4 \times 10^{-6}$, which we list at the bottom of Table 9 .

Combining this estimate with that of background/foreground star+planet pairs described previously, we arrive at a total blend frequency of $\mathrm{BF}=(12.7+4.4) \times 10^{-6} \approx 1.7 \times 10^{-5}$, which represents the a priori likelihood of a false positive. From a Bayesian point of view analogous to that adopted to validate previous Kepler candidates, our confidence in the planetary nature of K00137.03 will depend on how this likelihood compares to the a priori likelihood of a true transiting planet, addressed below.

The BF of $1.7 \times 10^{-5}$ corresponds to false-positive scenarios giving fits to the Kepler photometry that are within $3 \sigma$ of the best planet fit. We use a similar criterion to estimate the a priori transiting planet frequency by counting the KOIs in the Borucki et al. (2011b) sample that have radii within $3 \sigma$ of the value determined from the best fit to K00137.03 $\left(R_{p}=2.00 \pm 0.10 R_{\oplus}\right)$. We find 284 that are within this range, giving a planet frequency $\mathrm{PF}=284 / 156,453=1.8 \times 10^{-3}$.

This estimate does not account for the fact that the geometric transit probability of a planet is significantly increased by the presence of additional planets in the system (Kepler-18c and Kepler-18d in this case), given that mutual inclination angles in systems with multiple transiting planets have been found be relatively small (typically $1^{\circ}-4^{\circ}$; Lissauer et al. 2011b). Furthermore, a planet with the period of K00137.03 would be interior to the other two, further boosting the chances that it would transit. To incorporate this coplanarity effect, we have developed a Monte Carlo approach, described fully in the Appendix, in which we simulate randomly distributed reference planes and inclination dispersions around this plane, from which a weighted distribution of the inclination with respect to the line of sight for a third planet is calculated. Inclination angles relative to the random reference plane are assumed to follow a Rayleigh distribution (see Lissauer et al. 2011b). Although the known planets carry some information on the inclination dispersion, the probability of transit still depends somewhat on the allowed range of dispersion widths. When the assumed prior for the inclination dispersion is uniform up to $4^{\circ}$, following Lissauer et al. (2011b), we find that the flatness of the system results in a very significant increase in the transit probability for K00137.03 from $11.7 \%$ to $97 \%$. To be conservative, we adopt a larger range of possible inclination dispersions from $0^{\circ}$ to $10^{\circ}$, motivated by the upper limit from the similar Kepler-9 system (Holman et al. 2010). With this prior, the transit probability for K00137.03 becomes $84 \%$, or an increase by a factor of $\sim 7$ over the case of a single transiting planet.

Thus, the likelihood of a planet is more than 700 times greater $\left(\mathrm{PF} / \mathrm{BF}=0.013 / 1.7 \times 10^{-5} \approx 700\right)$ than that of a false positive, which we consider sufficient to validate K00137.03 as a true planet with a high degree of confidence. We designate this planet Kepler-18b. We note that our PF calculation assumes that the 1235 candidates cataloged by Borucki et al. (2011b) are all true planets. If we were to suppose conservatively that as many as $50 \%$ are false positives (an unlikely proposition that is also inconsistent with other evidence; see Borucki et al. 2011b; Howard et al. 2010), the planet likelihood would still be $\sim 350$ times greater than the likelihood of a blend, implying a false alarm rate sufficiently small to validate the candidate.

\section{PHYSICAL PROPERTIES OF THE PLANETS}

The Kepler-18 system consists of two low-density Neptunemass planets near a 2:1 mean-motion resonance and an inner super-Earth size planet. Its architecture bears a strong resemblance to Kepler-9, except that the Kepler-18 system is less compact and its planets are less dense. The use of the observed transit times as well as the RV data in the dynamical model of the Kepler-18 system places tight limits on the allowed planetary masses. We adopt these values as our best determination of the masses of the transiting planets in the Kepler-18 system. The last line in Table 8 gives the planet densities, computed from the final adopted planet masses. The TTV measurements together with the radial velocities restrict the masses of Kepler-18c $\left(17.3 \pm 1.9 M_{\oplus}\right)$ and Kepler-18d $\left(16.4 \pm 1.4 M_{\oplus}\right)$ to be similar to each other. Both are slightly lower than the mass of Neptune. Their radii, however, are $40 \%$ and $80 \%$ larger than Neptune, respectively, giving them bulk densities of $0.59 \pm 0.07 \mathrm{~g} \mathrm{~cm}^{-3}$ and $0.27 \pm 0.03 \mathrm{~g} \mathrm{~cm}^{-3}$, which are only 0.36 and 0.16 that of Neptune. The mass of Kepler-18b from the joint dynamical solution to the transit times and the RV measurements is $6.9 \pm 3.4 M_{\oplus}$. With its super-Earth-size radius of $2.00 \pm 0.10 R_{\oplus}$, the density of this inner planet in the system is $4.9 \pm 2.4 \mathrm{~g} \mathrm{~cm}^{-3}$.

Using the methods described in Miller et al. (2009) and Miller $\&$ Fortney (2011), we have modeled the thermal evolution and interior structure of the two "Neptune-class" planets, Kepler$18 \mathrm{c}$ and Kepler-18d. Both planets have inflated radii compared to Uranus and Neptune, which points to two effects. The first is that the high incident flux slows their contraction. The second is that the mass fraction of heavy element within these two planets is lower than that of Uranus and Neptune, meaning the mass fraction of $\mathrm{H}-\mathrm{He}$ gas is larger. Uranus and Neptune are $\sim 80 \%-90 \%$ heavy elements (e.g., water and rock) by mass (Fortney \& Nettelmann 2010), while below we show that the heavy element mass fractions of Kepler-18c and Kepler-18d are somewhat lower than these values.

Thermal evolution/contraction models are constrained such that the radius of each planet must be reproduced at the system's estimated age. As in Miller \& Fortney (2011), all relevant uncertainties are accounted for. These include uncertainties in the age of the system, the semimajor axes, masses, and radii of the planets, and the distribution of the heavy elements within each planet. We do not include an internal heating contribution due to eccentricity damping in either planet, as this power source is expected to fall off as $a^{-15 / 2}$ (Jackson et al. 2008) and, furthermore, the eccentricities suggested by the TTV solutions 

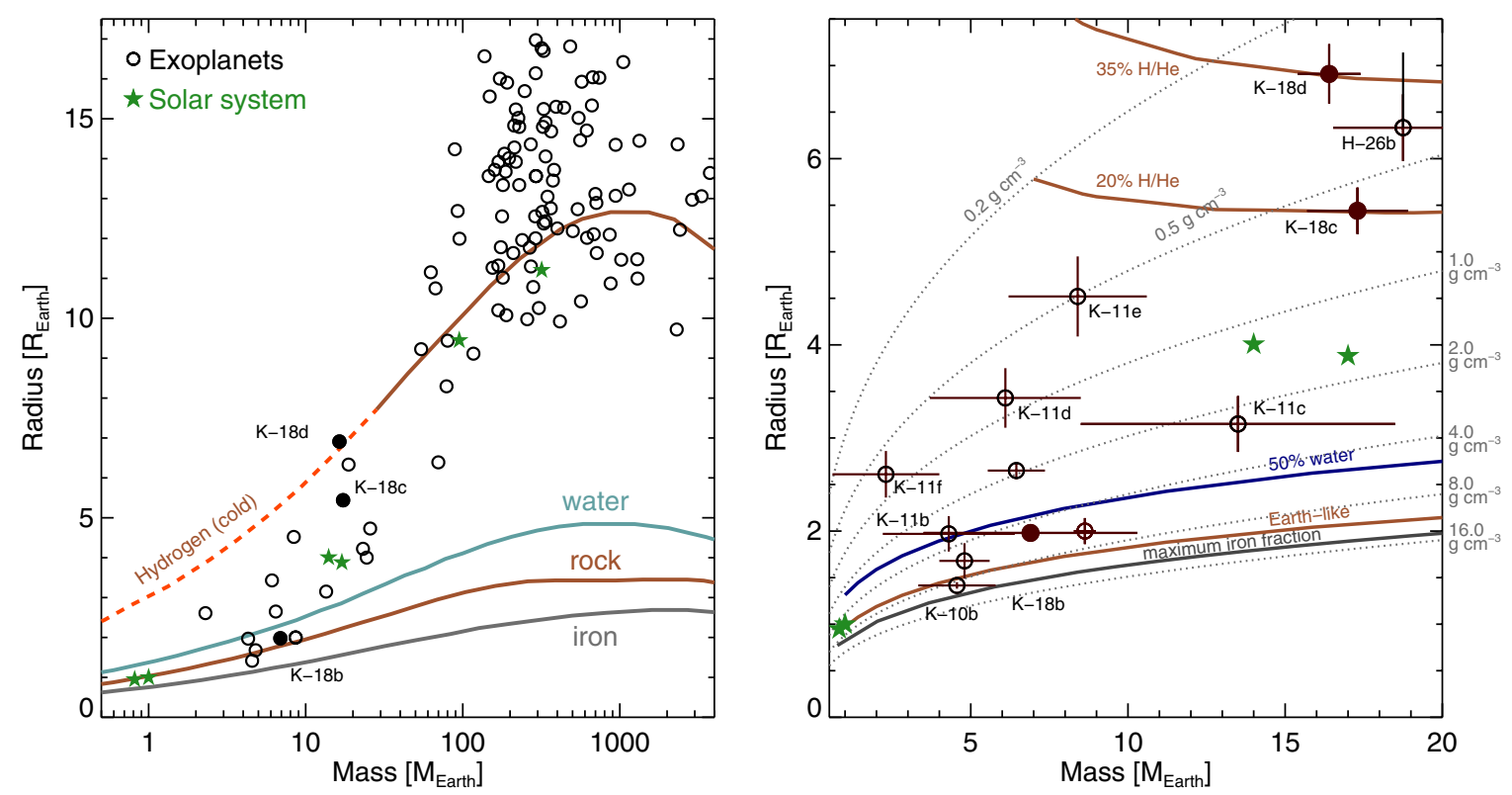

Figure 12. Mass-radius diagram for transiting exoplanets (circles) and solar system planets (stars). Left panel: known transiting exoplanets (open circles) and Kepler-18b, c, and d (filled circles), with curves of theoretical relations for cold pure hydrogen, water, rock (silicate), and iron (after Seager et al. 2007; Fortney et al. 2007). Two detailed models with mixtures and surface temperatures appropriate for Kepler-18b and Kepler-18c are also shown (Miller \& Fortney 2011). Right panel: zoom on the smaller planets, with curves of constant mean density and curves of detailed interior models of constant composition. The theoretical models are (from top to bottom): "Neptune-class" models with hydrogen/helium envelopes and 50-50 ice-rock (by mass) cores from thermal evolution calculations (Miller \& Fortney 2011) - the labeled $\mathrm{H} / \mathrm{He}$ fractions are averages; if all metals are in a core, these fractions will be $31 \%$ and $16 \%$, respectively; if mixed partially, they become $38 \%$ and $23 \%$, respectively, for the curves shown; the " $50 \%$ water" models have compositions of $44 \%$ silicate mantle and $6 \%$ iron core, and the nominal "Earth-like" composition with terrestrial iron/silicon ratio and no volatiles are by Valencia et al. (2006) and Zeng \& Sasselov (2011). The maximum mantle stripping limit (maximum iron fraction, minimum radius) was computed by Marcus et al. (2010). All these model curves of constant composition are for illustration purposes, as the degeneracy between composition mixtures and mean density is significant in this part of the mass-radius diagram. The data for the exoplanets were taken from Queloz et al. (2009); Charbonneau et al. (2009); Hartman et al. (2011); Batalha et al. (2011); Lissauer et al. (2011a); and Winn et al. (2011). We note that new analysis of CoRoT-7b (Hatzes et al. 2011) places it at a similar mass to Kepler-18b, and similar high-density composition to Kepler-10b. The three unmarked exoplanets surrounding Kepler-18b in the diagram are (in increasing mass) CoRoT-7 b, GJ 1214 b, and 55 Cnc e.

(A color version of this figure is available in the online journal.)

are quite small. A $50-50$ by mass ice-rock equation of state is used for the heavy elements. We find a heavy element mass of $13.5 \pm 1.8 M_{\oplus}$ in Kepler-18c ( $\sim 80 \%$ of the planet's mass) and $10.1 \pm 1.4 M_{\oplus}$ in Kepler-18d ( $\sim 60 \%$ of the planet's mass). Kepler-18c is clearly more "metal-rich" than Kepler-18d. Both planets are consistent with a core-accretion formation scenario in which $\sim 10 M_{\oplus}$ of heavy elements gravitationally captures an envelope of $\mathrm{H}-\mathrm{He}$ gas. This envelope itself may be enhanced in heavy elements, as is inferred for Uranus and Neptune.

Planet Kepler-18d, with a large radius of nearly $7 R_{\oplus}$, may point to a population of Neptune-mass exoplanets having relatively low heavy element mass fractions and radii approaching that of the gas giant regime. They appear very similar to HAT-P$26 \mathrm{~b}$, a 4.2 day planet orbiting a cooler K1-dwarf. The formation and evolution of such lower-density Neptune-class planets was recently studied in detail by Rogers et al. (2011). They find that modestly more massive $\mathrm{H} / \mathrm{He}$ envelopes than found on Uranus and Neptune (leading to larger planetary radii and lower densities) may be a common outcome of the core-accretion planet formation process.

The inner, 3.5 day period planet Kepler-18b, is a superEarth that requires a dominant mixture of water ice and rock, and no hydrogen/helium envelope. While the latter cannot be excluded simply on the basis of the planet's mass and radius, the evaporation timescale for a primordial $\mathrm{H} / \mathrm{He}$ envelope for a hot planet such as Kepler- $18 \mathrm{~b}$ is much shorter than the old age derived for the Kepler-18 system, and such a $\mathrm{H} / \mathrm{He}$ envelope should not be present. Thus, despite its lower equilibrium temperature, Kepler-18b resembles $55 \mathrm{Cnc}$ e and CoRoT- $7 \mathrm{~b}$ (as originally measured by Queloz et al. (2009), though the Hatzes et al. (2011) re-analysis makes CoRoT-7b very similar to Kepler-10b). Kepler-18b, together with $55 \mathrm{Cnc}$ e (Winn et al. 2011), are likely our best known cases yet of water planets with substantial steam atmospheres (given their high surface temperatures).

It is interesting to compare the three transiting planets in Kepler-18 in terms of their apparent compositions and orbital sequence. Kepler-18 reinforces a pattern already seen in Kepler-11, and with less confidence in Kepler-10 and Kepler9. Namely, inner planets are denser, though not always by very much - compare Kepler-18b versus c and d, and Kepler- $11 \mathrm{~b}$ and $\mathrm{c}$ versus $\mathrm{d}$, e, and $\mathrm{f}$ (Figure 12). It remains unclear at present whether this reflects a density gradient at formation or could be accomplished by evaporation later.

Kepler was competitively selected as the tenth Discovery mission. Funding for the Kepler Mission is provided by NASA's Science Mission Directorate. We are deeply grateful for the very hard work of the entire Kepler team. This research is based in part on observations made with the Spitzer Space Telescope, which is operated by the Jet Propulsion Laboratory, California Institute of Technology under a contract with NASA. Support for this work was provided by NASA through an award issued by JPL/Caltech. Some of the data presented herein were obtained at the W. M. Keck Observatory, which is operated as a scientific partnership among the California Institute of Technology, the University of California, and the National Aeronautics and Space Administration. The Keck Observatory 
was made possible by the generous financial support of the W. M. Keck Foundation.

\section{APPENDIX \\ INCORPORATING COPLANARITY IN MULTI-TRANSITING SYSTEMS TO ESTIMATE TRANSIT PROBABILITY}

In a system with $N$ transiting planets, what is the geometric probability that an additional planet (called "planet $N+1$ ") with a given period would transit, taking into account the fact that planetary orbits are expected (or observed) to be nearly coplanar? By incorporating the effect of coplanarity, the probability is significantly increased for additional planets to transit in known transiting planet systems compared to isotropically distributed planets.

In a one-planet, one-candidate system, $N=1$ and there is not much that can be done unless a prior assumption for the true mutual inclination is taken (Beatty \& Seager 2010). While in some cases, the true mutual inclination can be directly measured (see Ragozzine \& Holman 2010 for various methods), generally it will have to be inferred from population statistics. Lissauer et al. (2011b) estimate that the inclination distribution of shortperiod planetary systems seen by Kepler ranges from $1^{\circ}$ to $4^{\circ}$. This range could be used as a prior, both in the case of $N=1$ and in the case of higher multiplicities. Additionally, with careful analysis, the absence of transit timing and duration variations can be used to put an upper limit on mutual inclinations, as in the case of Kepler-9 where the mutual inclination must be less than $10^{\circ}$ (Holman et al. 2010).

However, systems with two or more transiting planets, such as Kepler-18, have evidence of being thin without direct reference to the greater population of Kepler multiples. Furthermore, the inclinations of the known planets themselves give information on both the location of the reference plane of that system (i.e., the Laplace plane) and the typical inclination dispersion. If both planets have the same inclination slightly different from $90^{\circ}$, as in the case of Kepler-18c and d, then this suggests that the reference plane for this system is something like the average plane between the two planets and that the inclinations with respect to that plane are likely quite small. In this situation, candidates with periods smaller than the inner planet are quite likely to transit (Ragozzine \& Holman 2010). If both planets have quite different inclinations, as in the case of Kepler-10 b and Kepler-10 c (Batalha et al. 2011), this implies an uncertainty in the location of the reference plane and a non-flat system, and the probability of additional planet transiting is enhanced over completely isotropic systems, but not as much (Fressin et al. 2011).

The goal is to quantify this effect in a natural way that uses all the available information. This is done using a Monte Carlo simulation that randomly generates reference planes, inclination dispersions, and nodal angles. The Monte Carlo trials that result in systems that match the observed inclinations of the $N$ planets well are given higher weight than the vast majority of trials that do a very poor job. The line-of-sight inclination of the additional planet is also calculated in each Monte Carlo trial, allowing for the generation of a final weighted distribution. The assumptions made in this calculation are that exoplanet reference planes are, a priori, randomly oriented in an isotropic way and that the inclinations (with respect to the reference plane) of all the planets in a system are faithfully represented by a single Rayleigh distribution. The latter assumption does not account for the possible anticorrelation between size and inclination that can occur in dynamically thermalized systems, i.e., larger planets may be more well aligned than smaller planets. With the Monte Carlo nature of the calculation and the error bars on inclinations that are typically produced by Kepler, the importance of a possible anticorrelation is reduced.

In practical terms, the Monte Carlo simulation draws a random inclination $\left(i_{r}\right)$ for the reference plane (uniform in $\cos i_{r}$ ). The width of the Rayleigh distribution $\sigma_{i}$ is drawn randomly from a distribution that is assumed a priori. For each Monte Carlo trial, a random inclination is drawn from the Rayleigh distribution as is a random nodal angle for each of the $N+1$ planets; the nodal angles are chosen uniformly between 0 and $2 \pi$. Using the method in Equation (2) of Ragozzine \& Holman (2010), the on-the-sky inclinations of the $N$ planets are calculated. These are compared to the observed inclinations by computing the standard Gaussian $z$-score, i.e., by calculating the number of standard deviations away the trial value is from the observed value. (This could be modified if the inclinations and errors of the known planets are either not known or are known not to be Gaussian.) The assigned weight for each planet is equal to the area under the Gaussian that has more extreme scores than the trial value, i.e., with a $z$-score of $z=i_{\mathrm{MC}}-i_{\mathrm{obs}} / i_{\mathrm{err}}$, the weight is $w=1.0-\operatorname{erf}(|z| / \sqrt{2})$, where $i_{\mathrm{MC}}$ is the calculated trial inclination, $i_{\mathrm{obs}}$ is the observed inclination with error $i_{\mathrm{err}}$, and erf is the standard error function. If the trial value of the inclination is exactly the same as the observed inclination, the weight is 1 ; if it is many standard deviations off, then the weight is essentially 0 . The total weight for a Monte Carlo trial is the product of these weights over all the known $N$ planets based on their inclinations. As expected, the weight increases when the reference plane is taken near the average of the known planetary inclinations with a Rayleigh width similar to the standard deviation between the known inclinations.

Each of the Monte Carlo trials also calculates an inclination for planet $N+1$, based on the same reference inclination and $\sigma_{i}$. Using the weights derived from the observed inclinations of the $N$ transiting planets, the weighted distribution of the inclination of planet $N+1$ can be created. To calculate the probability that planet $N+1$ would be found transiting, the sum of the weights for those simulations that would have produced a transiting planet is divided by the sum of the weights for the entire Monte Carlo simulation.

Despite the information on the inclination dispersion from the known planets that is included in the weighting, the answer depends somewhat on the prior assumption for $\sigma_{i}$. For example, when applied to Kepler-18 with Kepler-18b as planet $N+1$, this technique predicts a transit probability of $47 \%, 84 \%$, and $97 \%$ when the prior on $\sigma_{i}$ is uniformly drawn between $0^{\circ}$ and $90^{\circ}, 0^{\circ}$ and $10^{\circ}$, and $0^{\circ}$ and $4^{\circ}$, respectively. In every case, the near coplanarity and low impact parameter of the two outer planets significantly increase the probability that Kepler- $18 \mathrm{~b}$ is transiting. Changing the prior on the inclination of the reference plane does not affect the result. This method provides a quantitative way to estimate the increased in-transit probability for planets in multi-transiting systems. When applied in combination with BLENDER and other techniques, it will allow multi-transiting systems to be validated more easily than singly transiting systems. Note that the method described here only estimates the improvement in the probability of the planet hypothesis due to geometric constraints, and does not include the additional effect that planets tend to be found in multiple systems, as discussed in Lissauer et al. (2011b). 


\section{REFERENCES}

Agol, E., Steffen, J., Sari, R., \& Clarkson, W. 2005, MNRAS, 359, 567

Ballard, S., Christiansen, J. L., Charbonneau, D., et al. 2010, ApJ, 716, 1047

Batalha, N. M., Borucki, W. J., Bryson, S. T., et al. 2011, ApJ, 729, 27

Batalha, N. M., Borucki, W. J., Koch, D. G., et al. 2010a, ApJ, 713, L109

Batalha, N. M., Rowe, J. F., Gilliland, R. L., et al. 2010b, ApJ, 713, L103 Beatty, T. G., \& Seager, S. 2010, ApJ, 712, 1433

Beerer, I. M., Knutson, H. A., Burrows, A., et al. 2011, ApJ, 727, 23

Borucki, W. J., Koch, D., Basri, G., et al. 2010a, Science, 327, 977

Borucki, W. J., Koch, D. G., Basri, G., et al. 2011a, ApJ, 728, 117

Borucki, W. J., Koch, D. G., Brown, T. M., et al. 2010b, ApJ, 713, L126

Borucki, W. J., Koch, D. G., Basri, G., et al. 2011b, ApJ, 736, 19

Brown, T. M., Latham, D. W., Everett, M. E., \& Esquerdo, G. A. 2011, AJ, 142, 112

Brugamyer, E., Dodson-Robinson, S. E., Cochran, W. D., \& Sneden, C. 2011, ApJ, 738, 97

Bryson, S. T., Tenenbaum, P., Jenkins, J. M., et al. 2010, ApJ, 713, L97

Butler, R. P., Marcy, G. W., Williams, E., et al. 1996, PASP, 108, 500

Caldwell, D. A., Kolodziejczak, J. J., Van Cleve, J. E., et al. 2010, ApJ, 713, L92

Charbonneau, D., Allen, L. E., Megeath, S. T., et al. 2005, ApJ, 626, 523

Charbonneau, D., Berta, Z. K., Irwin, J., et al. 2009, Nature, 462, 891

Cochran, W. D., Hatzes, A. P., \& Paulson, D. B. 2002, AJ, 124, 565

Deming, D., Knutson, H., Agol, E., et al. 2011, ApJ, 726, 95

Désert, J.-M., Charbonneau, D., Fortney, J. J., et al. 2011a, ApJ, submitted (arXiv:1102.0555)

Désert, J.-M., Lecavelier des Etangs, A., Hébrard, G., et al. 2009, ApJ, 699, 478

Désert, J.-M., Sing, D., Vidal-Madjar, A., et al. 2011b, A\&A, 526, A12

Dunham, E. W., Borucki, W. J., Koch, D. G., et al. 2010, ApJ, 713, L136

Eastman, J., Siverd, R., \& Gaudi, B. S. 2010, PASP, 122, 935

Fabrycky, D. C. 2010, in Exoplanets, ed. S. Seager (Tucson, AZ: Univ. Arizona Press), 217

Fazio, G. G., Hora, J. L., Allen, L. E., et al. 2004, ApJS, 154, 10

Fortney, J. J., Marley, M. S., \& Barnes, J. W. 2007, ApJ, 659, 1661

Fortney, J. J., \& Nettelmann, N. 2010, Space Sci. Rev., 152, 423

Fressin, F., Torres, G., Desert, J.-M., et al. 2011, ApJ, in press (arXiv:1105.4647)

Gilliland, R. L., Jenkins, J. M., Borucki, W. J., et al. 2010, ApJ, 713, L160

Gregory, P. C. 2011, MNRAS, 410, 94

Halbwachs, J. L., Mayor, M., Udry, S., \& Arenou, F. 2003, A\&A, 397, 159

Hartman, J. D., Bakos, G. Á., Kipping, D. M., et al. 2011, ApJ, 728, 138

Hatzes, A. P., Fridlund, M., Nachmani, G., et al. 2011, ApJ, in press (arXiv:1105.3372)

Hayward, T. L., Brandl, B., Pirger, B., et al. 2001, PASP, 113, 105

Holman, M. J., Fabrycky, D. C., Ragozzine, D., et al. 2010, Science, 330, 51

Horch, E. P., Gomez, S. C., Sherry, W. H., et al. 2011, AJ, 141, 45

Howard, A. W., Marcy, G. W., Johnson, J. A., et al. 2010, Science, 330, 653

Howell, S. B., Everett, M. E., Sherry, W., Horch, E., \& Ciardi, D. R. 2011, AJ, 42, 19

Jackson, B., Greenberg, R., \& Barnes, R. 2008, ApJ, 681, 1631

Jenkins, J. M., Borucki, W. J., Koch, D. G., et al. 2010a, ApJ, 724, 1108
Jenkins, J. M., Caldwell, D. A., Chandrasekaran, H., et al. 2010b, ApJ, 713, L120

Jenkins, J. M., Caldwell, D. A., Chandrasekaran, H., et al. 2010c, ApJ, 713, L87

Jenkins, J. M., Chandrasekaran, H., McCauliff, S. D., et al. 2010d, Proc. SPIE, 7740, 77400D

Johnson, J. A., Winn, J. N., Albrecht, S., et al. 2009, PASP, 121, 1104

Knutson, H. A., Charbonneau, D., Allen, L. E., Burrows, A., \& Megeath, S. T. 2008, ApJ, 673, 526

Koch, D. G., Borucki, W. J., Basri, G., et al. 2010a, ApJ, 713, L79

Koch, D. G., Borucki, W. J., Rowe, J. F., et al. 2010b, ApJ, 713, L131

Latham, D. W., Borucki, W. J., Koch, D. G., et al. 2010, ApJ, 713, L140

Lissauer, J. J., Fabrycky, D. C., Ford, E. B., et al. 2011a, Nature, 470, 53

Lissauer, J. J., Ragozzine, D., Fabrycky, D. C., et al. 2011b, ApJ, in press (arXiv:1102.0543)

Mandel, K., \& Agol, E. 2002, ApJ, 580, L171

Marcus, R. A., Sasselov, D., Hernquist, L., \& Stewart, S. T. 2010, ApJ, 712, L73

Marcy, G. W., Butler, R. P., Vogt, S. S., et al. 2008, Phys Scr. T, 130, 14001

Markwardt, C. B. 2009, in ASP Conf. Ser. 411, Astronomical Data Analysis Software and Systems XVIII, ed. D. A. Bohlender, D. Durand, \& P. Dowler (San Francisco, CA: ASP), 251

Mazeh, T. 2008, in Tidal Effects in Stars, Planets and Disks, ed. M.-J. Goupil \& J.-P. Zahn (EAS Publication Series, Vol. 29; Paris: EDP), 1

Miller, N., \& Fortney, J. J. 2011, ApJ, 736, L29

Miller, N., Fortney, J. J., \& Jackson, B. 2009, ApJ, 702, 1413

Miralda-Escudé, J. 2002, ApJ, 564, 1019

Morton, T. D., \& Johnson, J. A. 2011, ApJ, 738, 170

Pont, F., Zucker, S., \& Queloz, D. 2006, MNRAS, 373, 231

Queloz, D., Bouchy, F., Moutou, C., et al. 2009, A\&A, 506, 303

Raghavan, D., McAlister, H. A., Henry, T. J., et al. 2010, ApJS, 190, 1

Ragozzine, D., \& Holman, M. J. 2010, ApJ, submitted (arXiv:1006.3727)

Robin, A. C., Reylé, C., Derrière, S., \& Picaud, S. 2003, A\&A, 409, 523

Rogers, L. A., Bodenheimer, P., Lissauer, J. J., \& Seager, S. 2011, ApJ, 738, 59

Seager, S., Kuchner, M., Hier-Majumder, C. A., \& Militzer, B. 2007, ApJ, 669, 1279

Sneden, C. A. 1973, PhD thesis, Univ. Texas at Austin

Steffen, J. H., Batalha, N. M., Borucki, W. J., et al. 2010, ApJ, 725, 1226

Torres, G., Fressin, F., Batalha, N. M., et al. 2011, ApJ, 727, 24

Torres, G., Konacki, M., Sasselov, D. D., \& Jha, S. 2004, ApJ, 614, 979

Troy, M., Dekany, R. G., Brack, G., et al. 2000, Proc. SPIE, 4007, 31

Valencia, D., O'Connell, R. J., \& Sasselov, D. 2006, Icarus, 181, 545

Valenti, J. A., \& Fischer, D. A. 2005, ApJS, 159, 141

Valenti, J. A., \& Piskunov, N. 1996, A\&AS, 118, 595

Vogt, S. S., Allen, S. L., Bigelow, B. C., et al. 1994, Proc. SPIE, 2198, 362

Werner, M. W., Roellig, T. L., Low, F. J., et al. 2004, ApJS, 154, 1

Winn, J. N., Matthews, J. M., Dawson, R. I., et al. 2011, ApJ, 737, L18

Wu, H., Twicken, J. D., Tenenbaum, P., et al. 2010, Proc. SPIE, 7740, 19

Yi, S., Demarque, P., Kim, Y.-C., et al. 2001, ApJS, 136, 417

Zechmeister, M., \& Kürster, M. 2009, A\&A, 496, 577

Zeng, L., \& Sasselov, D. 2011, PASP, submitted 